\title{
Topological quantum phase transitions and criticality in a longer-range Kitaev chain
}

\author{
Y. R. Kartik, ${ }^{1,2, *}$ Ranjith R. Kumar $\odot,,^{1,2, \dagger}$ S. Rahul, ${ }^{1,2, \dagger}$ Nilanjan Roy $\odot,{ }^{3,4, \S}$ and Sujit Sarkar ${ }^{1, \|}$ \\ ${ }^{1}$ Theoretical Sciences Division, Poornaprajna Institute of Scientific Research, Bidalur, Bengaluru 562164, India \\ ${ }^{2}$ Graduate Studies, Manipal Academy of Higher Education, Madhava Nagar, Manipal-576104, India \\ ${ }^{3}$ Department of Physics, Indian Institute of Science Education and Research, Bhopal, Madhya Pradesh 462066, India \\ ${ }^{4}$ Department of Physics, Indian Institute of Science, Bangalore 560012, India
}

(Received 12 February 2021; revised 23 May 2021; accepted 26 July 2021; published 10 August 2021)

\begin{abstract}
In an attempt to theoretically investigate the quantum phase transition and criticality in topological models, we study a Kitaev chain with longer-range couplings (finite number of neighbors) as well as truly long-range couplings (infinite number of neighbors). We carry out an extensive topological characterization of the momentum space to explore the possibility of obtaining higher order winding numbers and analyze the nature of their stability in the model. The occurrences of phase transitions from even-to-even and odd-to-odd winding numbers are observed with decreasing longer-rangeness in the system. We derive topological quantum critical lines and study them to understand the behavior of criticality. A suppression of higher order winding numbers is observed with decreasing longer-rangeness in the model. We show that the mechanism behind such phenomena is due to the superposition and vanishing of the topological quantum critical lines associated with the higher winding number. Through the study of the Berry connection, we show the possible different behaviors of critical lines when they undergo superposition along with the corresponding critical exponents. We analyze the behavior of the long-range models through the momentum space characterization. We also provide the exact solution for the problem and discuss the experimental aspects of the work.
\end{abstract}

DOI: 10.1103/PhysRevB.104.075113

\section{INTRODUCTION}

Topological states of matter are considered as novel phases of matter in modern physics. The concept started as a theoretical prediction and expanded towards experimental realizations $[1,2]$. For almost a century, Landau theory of spontaneous symmetry breaking explained almost all the phases of matter except topological phases [3-5]. Landau theory relies on the existence of the local order parameter, which is absent in topological state of matter [6]. This created a need for some alternate way to establish topological characterization. Topological invariant is a promising quantity, which explains gapped topological phases in a very accurate way, but it fails when comes to topological quantum phase transition (TQPT) [7].

TQPT is basically quantum phase transition at quantum critical points $(\mathrm{QCP})$, since they occur at zero temperature [8-10]. At QCP, instead of vanishing local order parameter, topological system has a special kind of diverging topological correlation factor in its electronic band structure [11]. This topological correlation factor is directly associated with topological invariant. Hence topological invariants are well quantized at the gapped phases and ill-defined at QCP. So far for a $1 \mathrm{D}$ system, winding number (WN) is the most accepted

\footnotetext{
*yrkartik@gmail.com

†ranjith.btd6@gmail.com

†rahulastronomer02@gmail.com

§nilanjanroy.physics@gmail.com

" sujit.tifr@gmail.com
}

form of topological invariant which is the integration of Berry connection (vector potential) over the Brillouin zone. Hence considering this form of the correlation factor, it is possible to extract the information around the QCP [12]. This insight helped scientific community to think about topological state of matter from the perspective of criticality. Even earlier there were many attempts like renormalization group [13-15], curvature function renormalization group [16] and other scaling approaches to explain TQPT $[17,18]$. But now it is evident that there is a possibility to explain criticality through correlation function, curvature function [16], critical exponents, and universality class of TQPT $[11,12]$.

Topological states of matter are the area of curiosity because of the emergence of exotic quasiparticles unlike fermions and bosons [19]. The area became more prominent with its real life applications [20]. It is possible to generate higher order localized edge modes through periodic driving and longer-range couplings, where the previous method yields dynamical localized modes and the later yields static modes $[21,22]$. Higher order localized modes have their own interests in topological state of matter. On the other hand, long-range topological models are the more generalized version of novel phases of matter [23]. This includes realization of new phases like edge insulating topological phases $[24,25]$ with fractional topological invariants [26] and quasiparticles like Majorana zero modes (MZM), massive majorana modes [27]. In this work, we carry out a theoretical study of a topological longerrange as well as truly long-range model. Our motivation is two folded: topological characterization of the momentum space and study of the quantum criticality for the long-range models. 
We theoretically study the topological quantum phase transition and criticality in Kitaev chain with "longer-range" couplings which are finite-ranged but more than nearestneighbors. Here we stick to the terminology used in the existing literature $[26,28]$. We derive topological quantum critical lines and find a suppression of higher order winding numbers with decreasing longer-rangeness in the model. As a reason behind this we argue for the superposition of two critical lines with different winding numbers followed by vanishing of the critical line with higher winding number. We analyze the possibilities of obtaining higher order winding numbers and study their stability in the model. Our analysis shows the phase transition from even-to-even and odd-to-odd winding numbers with decreasing longer-rangeness in the system. We also provide a few exact solutions for winding number.

This paper is organized as follows. In Sec. II, we explain our model Hamiltonian and aim of the study. In Sec. III, we carry out the topological characterization of momentum space by calculating winding number. We also obtain the phase diagram with a detailed study of the critical lines. In Sec. IV, We elaborate on the topological quantum criticality of our longer-range system and extend it with respect to stability of higher order winding numbers. We perform momentum space characterization to explain the behavior of long-range models. In Sec. V, we study the parameter space in order to understand critical phases with a few relevant exact solutions. In Sec. VI, we provide the outlook and experimental aspects of the work. Then we conclude in Section VII.

\section{THE MODEL AND AIM OF THE STUDY}

We consider 1D Kitaev model with $r$ neighboring interactions (both hopping and pairing) [26]. This kind of model for infinitely long-range model was studied in Ref. [23] and for longer-range Kitaev chain was studied in Ref. [26]. This model helps to understand the emergence and behavior of Majorana modes and topological invariant in the longer-range as well as in the truly long-range systems. We define our model Hamiltonian as

$$
\begin{aligned}
H= & -\sum_{j=1}^{L} \mu\left(c_{j}^{\dagger} c_{j}-1 / 2\right) \\
& -\sum_{j=1}^{L-l} \sum_{l=1}^{r}\left(J_{l} c_{j}^{\dagger} c_{j+l}+\Delta_{l} c_{j}^{\dagger} c_{j+l}^{\dagger}+\text { H.c. }\right),
\end{aligned}
$$

where $\mu$ is the chemical potential, $L$ is the number of lattice sites, $J_{l}$ and $\Delta_{l}$ are the strengths of hopping and pairing terms respectively with long-range interactions of the form

$$
J_{l}=\frac{J_{0}}{d_{l}^{\alpha}}, \Delta_{l}=\frac{\Delta_{0}}{d_{l}^{\beta}} .
$$

These hopping and pairing terms couple the lattice site $j$ with $j+l$. For a system with open boundary condition, the distance $d_{l}=l$. For closed boundary, $d_{l}=\min (l, L-l)$. $\alpha$ and $\beta$ are the non-negative parameters which represent the power-law decay of hopping and pairing terms, respectively. When $\alpha \rightarrow \infty$, system behaves as a Kitaev chain with only long-range pairing and when $\beta \rightarrow \infty$, system behaves as a Kitaev chain with only long- range hopping. When both $\alpha, \beta \rightarrow \infty$ system behaves as original Kitaev chain $[19,23,26]$.

After a Fourier transformation, one can write the model in the momentum space as

$$
\begin{aligned}
H= & \sum_{k>0}\left(-\mu-2 J_{0} \sum_{l=1}^{r} \frac{\cos [k l]}{l^{\alpha}}\right)\left(\psi_{k}^{\dagger} \psi_{k}+\psi_{-k^{\dagger}}^{\dagger} \psi_{-k}\right) \\
& +2 i \Delta_{0} \sum_{k>0} \sum_{l=1}^{r}\left(\frac{\sin [k l]}{l^{\beta}}\right)\left(\psi_{k}^{\dagger} \psi_{-k^{\dagger}}+\psi_{k} \psi_{-k}\right)
\end{aligned}
$$

where $\psi^{\dagger}(k)(\psi(k))$ is the creation (annihilation) operator of the spinless fermion of momentum $k$. We can write the BdG Hamiltonian as

$$
H_{B d G}(k)=\left(\begin{array}{cc}
\chi_{z}(k) & i \chi_{y}(k) \\
-i \chi_{y}(k) & -\chi_{z}(k)
\end{array}\right) .
$$

We can express the Hamiltonian by Anderson pseudospin approach [29,30]. One can write the BdG Hamiltonian in the pseudospin basis as

$$
H_{B d G}(k)=\chi_{x}(k) \vec{\tau}_{1}+\chi_{y}(k) \vec{\tau}_{2}+\chi_{z}(k) \vec{\tau}_{3},
$$

where $\tau_{i}=\left(\tau_{1}, \tau_{2}, \tau_{3}\right)$ are the Pauli matrices in particle-hole space and the coefficients are

$$
\begin{aligned}
& \chi_{x}(k)=0, \\
& \chi_{y}(k)=2 \Delta_{0} \sum_{l=1}^{r} \frac{\sin [k l]}{l^{\beta}}, \\
& \chi_{z}(k)=\left(-\mu-2 J_{0} \sum_{l=1}^{r} \frac{\cos [k l]}{l^{\alpha}}\right) .
\end{aligned}
$$

It is to be noted that for $r \rightarrow \infty$ the series involving $\frac{\cos (k l)}{l^{\alpha}}$ and $\frac{\sin (k l)}{l^{\beta}}$ terms give rise to polylogarithmic functions $[23,26]$. The quasiparticle excitation energy is given by

$$
E_{k}= \pm \sqrt{\left(\chi_{z}(k)\right)^{2}+\left(\chi_{y}(k)\right)^{2}} .
$$

In the current work, our interest is to analyze the Kitaev model with finite number of interacting neighbors. We consider the Hamiltonian with longer-range hopping and pairing up to finite $r$ neighbors with $\alpha=\beta$ and $J_{0}=\Delta_{0}=\lambda$, so that within this regime the system resembles isotropic Kitaev chain with $r$ neighboring interactions. Hence as one varies the value of $r$, it is possible to generate Kitaev chain whose neighboring terms have a power law decay in the associated couplings.

Here we explain the aim of our work. (i) For a longerrange Kitaev model with $r$ nearest neighbors, there exists $r$ topological phases $[21,26]$ and one can recover original Kitaev chain when $\alpha, \beta \rightarrow \infty,[19,23]$. It is a very effective method to choose more number of neighbors to achieve higher order WNs. Here our interest is to understand and analyze the possibility of obtaining all the $r$ topological phases in an isotropic Kitaev chain. (ii) For a transition between topological phases of higher $\mathrm{WN}$ and lower $\mathrm{WN}$, there may exist a staircase of TQPTs [21]. Here we attempt to carry out an analysis to extract the order of such TQPTs and study the stability of these high-WN topological phases. (iii) For a longer-range model with $r$ interacting neighbors, there exist 
$r$ topological phases and critical lines which distinguish the topological phases [28]. Hence, when a longer-range model is reduced to its original short-range version, there may be a change in the behavior of its corresponding critical lines also. To explain this, we derive all possible critical lines and study their behavior from the perspective of quasiparticle energy spectrum, curvature function and ground-state energy. (iv) In a truly long-range model, there may exist some emergent quasiparticles-like massive edge modes, based on the selection of parameter space. However, we try to analyze this phenomenon from the perspective of momentum space characterization.

\section{TOPOLOGICAL CHARACTERIZATION IN MOMENTUM SPACE AND TOPOLOGICAL QUANTUM CRITICALITY}

Winding number is the most accepted form of topological invariant $[31,32]$. In this section, we derive $\mathrm{WN}$ for different choices of parameters to understand the possible topological index of the system. We explore the superposition and vanishing of TQCLs through the study of Berry connection and ground-state energy. We study and analyze the ground-state energy to explain the stability of higher order WNs [21,33$35]$. We study the parameter space through pseudospin vectors and also derive quite a few exact solutions for the WN.

\section{A. Winding number}

For a system in $1 \mathrm{D}, \mathrm{WN}$ is defined as

$$
W=\frac{1}{2 \pi} \oint \frac{\partial \theta_{k}}{\partial k} d k=\frac{1}{2 \pi} \oint \frac{\chi_{z} \partial_{k} \chi_{y}-\chi_{y} \partial_{k} \chi_{z}}{\chi_{z}^{2}+\chi_{y}^{2}} d k,
$$

where $\theta_{k}=\tan ^{-1}\left(\frac{x_{y}}{\chi_{z}}\right)$. This relation holds good even for longer-range models with $r$ nearest interacting neighbors, although this definition of $W$ is ill-defined at TQPT. In all possible gapped phases, topological index secures a quantized value (integers like $W=0,1,2,3, \ldots, r$ ) and this depends on the number of interacting neighbors. This is because, the $\mathrm{WN}$ is always associated with the modulo of $2 \pi$. The phase $W=0$ represents nontopological phase. One can achieve higher order WNs by increasing the number of interacting neighbors. The transition from one topological phase to other occurs through topological quantum critical lines (TQCL) [30]. These TQCL are the gap closing points in the quasienergy spectrum. It is important to note that, for all TQCLs there are the gap closings but all the gap closings need not be TQCLs [16]. The gap closing results in the QPT and if this QPT differentiates two distinct topological phases, then this gap closing points are known as topological quantum phase transition (TQPT) points.

WN always corresponds to the number of localized edge modes of the topological gapped phases. Recently there are some works which show the localized edge modes even at the criticality [36-49]. Hence it is clear and meaningful to find the WN around criticality and physically it is possible to find the corresponding edge modes. There are some cases, where one can get the fractional WNs at critical points. Even though, there are no proper experimental evidences for fractional edge modes, it is possible to define the fractional WN around criticality. The definition of WN for TQPT can be modified by excluding an infinitesimal neighborhood of the gapless/critical points. Then the modified expression for WN is given by [39]

$$
W=\frac{1}{2 \pi} \lim _{\delta \rightarrow 0} \int_{\forall i:\left|k-k_{i}\right|>\delta} \frac{\partial \theta_{k}}{\partial k} d k,
$$

where $\left\{k_{i}\right\}$ is the set of critical/gapless points in the momentum space. Thus, we can define the $\mathrm{WN}$ at and around the critical point.

Here we consider a limited number of interacting neighbors $r=2,3,4$ and obtain the possible topological phases with integer WNs. The TQPTs among these topological phases for $r=2,3,4$ are shown in following cases, respectively.

We also calculate all possible critical lines to understand the phase diagram of the system and its dependence on the decay parameter $\alpha$. In quantum systems, the transition occurs from one phase to another through the QCP, which are the gapless points in the excitation energy spectrum. The quasiparticle excitation energy spectrum of our model is given by Eq. (6).

\section{Case 1: when $r=2$}

Figure 1 shows the phase diagram for $r=2$. Here we can see the interaction is up to the second nearest neighbor in the chain and Hamiltonian is given by Eq. (1), with $r=$ $2, \lambda=\Delta=J$, and $\alpha=\beta$. Here, the gap closings occur at three different values of $k$. (Appendix) which corresponds to three different TQCLs.

For this $r=2, \mathrm{WN}$ is given by

$$
W=\frac{1}{2 \pi} \int_{-\pi}^{\pi} \frac{\left(\frac{4 \lambda^{2} Q A}{P^{2}}+\frac{2 \lambda B}{P}\right)}{\left(\frac{4 \lambda^{2} Q^{2}}{P^{2}}+1\right)} d k
$$

where

$$
\begin{aligned}
& A=\left(-2^{1-\alpha} \sin (2 k)-\sin (k)\right), \\
& B=\left(2^{1-\alpha} \cos (2 k)+\cos (k)\right), \\
& P=\left(-2 \lambda\left(2^{-\alpha} \cos (2 k)+\cos (k)\right)-\mu\right), \\
& Q=\left(2^{-\alpha} \sin (2 k)+\sin (k)\right)
\end{aligned}
$$

Figure 1(a) shows the $\mathrm{WN}$ for the case $r=2$. For $\alpha=$ $0,0.1$, one can observe transitions among $W: 0 \leftrightarrow 1$ and $W$ : $0 \leftrightarrow 2$. For $\alpha=0.5$, one can observe the transitions among $W: 0 \leftrightarrow 1, W: 2 \leftrightarrow 1$, and $W: 0 \leftrightarrow 2$. We note that the plateau of $W=2$ region for $\alpha=0.5$ is reduced as compared to that for $\alpha=0$. This indicates the decrease in the stability of the higher order $\mathrm{WN}$ as $\alpha$ increases. For $\alpha=0.9$, the $W=2$ topological phase has very short plateau and for $\alpha=1,1.1$, it is clearly seen that $W=2$ region is absent and we observe only $W=1$ and $W=0$ phases similar to the original Kitaev chain.

Here we have three TQCLs, i.e., the red (first TQCL for $k=0$ ), blue (second TQCL for $k=\pi$ ), and green lines [third TQCL for $k=\cos ^{-1}\left(-2^{\alpha-1}\right)$ ] (see Appendix). Throughout the case first TQCL (red) separates $W=1$ and $W=0$ and it is unaltered with variation of $\alpha$. When $\alpha=0$, the second critical line (blue) lies on the $\lambda$ axis [Fig. 1(b1)]. As one 
(a) $\mu=1$

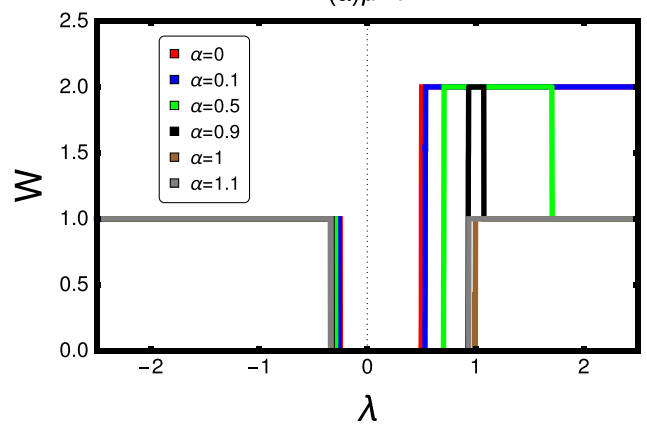

(b1) $\alpha=0$

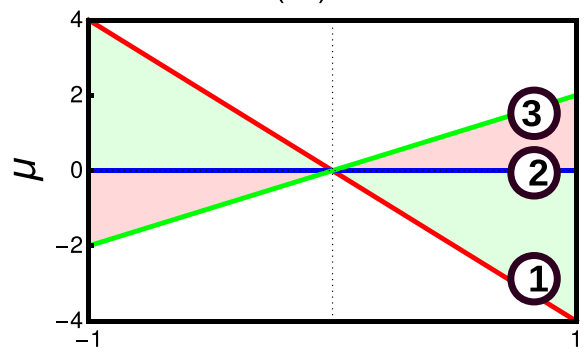

$\lambda$

(b4) $\alpha=0.9$

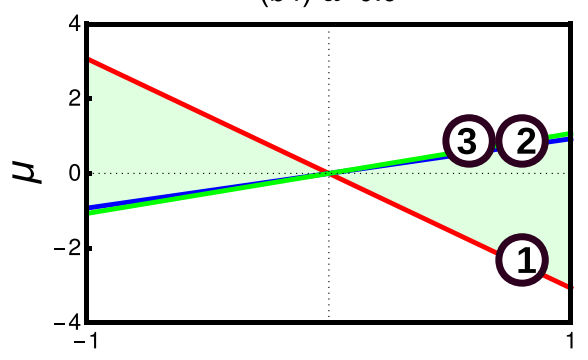

$\lambda$

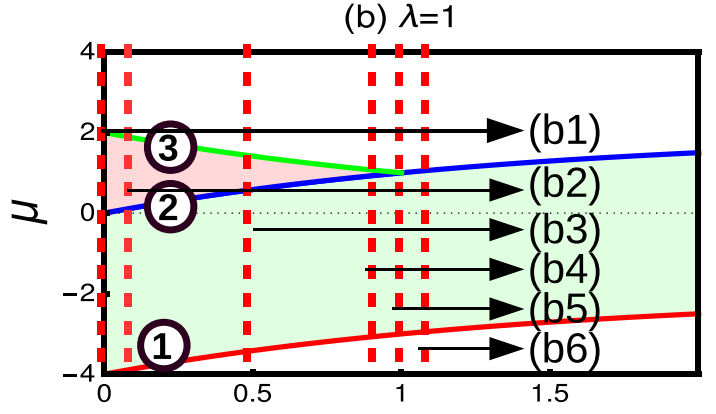

$\alpha$

(b2) $\alpha=0.1$

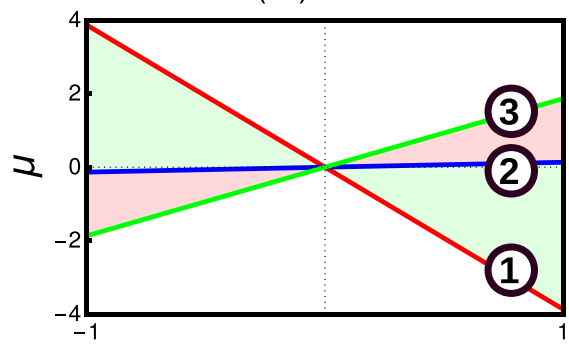

$\lambda$

(b5) $\alpha=1$

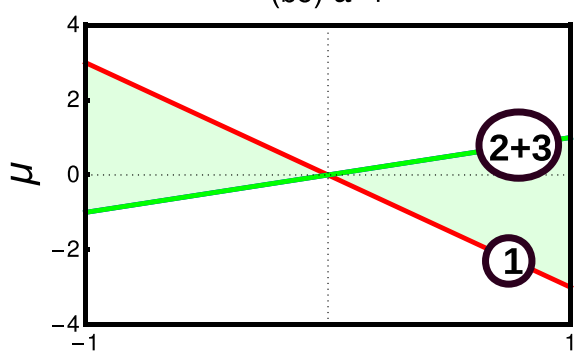

$\lambda$

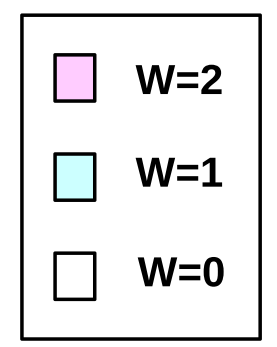

(b3) $\alpha=0.5$

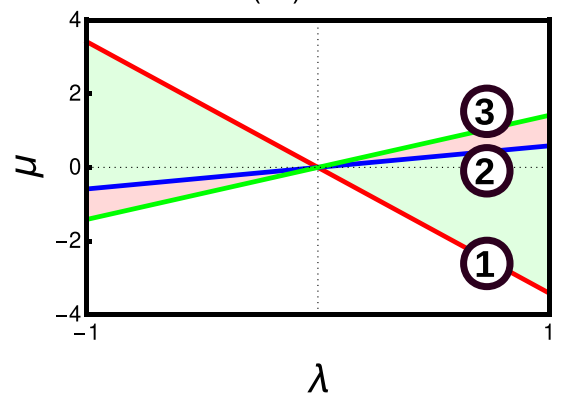

(b6) $\alpha=1.1$

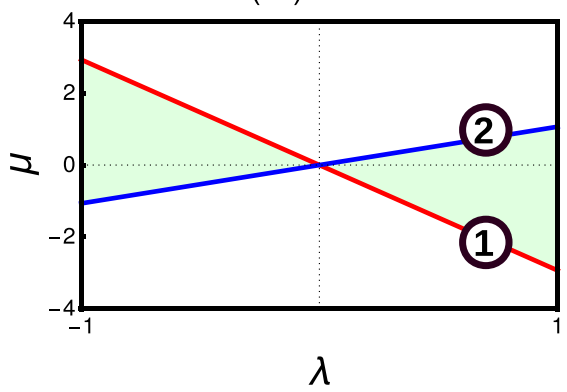

FIG. 1. Topological phase diagram of longer-range Kitaev chain with $r=2$ neighbors. (a) Winding number study with respect to $\lambda$ for different values of $\alpha$. (b) Behavior of topological phases in $\mu$ - $\alpha$ parameter space with fixed $\lambda$. [(b1)-(b6)] Corresponding topological phase diagrams of model in $\mu-\lambda$ parameter space with increasing values of $\alpha$. This way of representation helps to understand the behavior of TQCLs. Red, blue, and green lines represent the first, second, and third TQCLs, respectively. With the increasing value of $\alpha$, the second TQCL starts moving in anticlockwise manner to superpose with third TQCL. This causes the suppression of $W=2$ phase. The third TQCL vanishes for $\alpha>1$ and the model reduces to short-range Kitaev chain.

gradually increases $\alpha$ the second critical line starts moving in anticlockwise direction [Figs. 1(b2)-1(b4)] and superposes with third critical line (green) [Fig. 1(b5)]. This results in the vanishing of $W=2$ topological phase which in turn leads to vanishing of the third TQCL. We observe that the third TQCL vanishes for $\alpha>1$ [Fig. 1(b6)], which is consistent with the fact that the point $k=\cos ^{-1}\left(-2^{\alpha-1}\right)$ does not exist for $\alpha>1$. The second TQCL keeps on moving anticlockwise with further increase of $\alpha$ till $\alpha=1.5$ beyond which it stops moving with $\alpha$ indicating the limit of the original Kitaev chain.

\section{Case 2: when $r=3$}

Figure 2(a) shows the phase diagram for $r=3$ where we can see the interaction is up to the third nearest neighbor in the chain. Here gap closings occur at four different values of $k$ (Appendix), which corresponds to four different TQCLs. Here the Hamiltonian is given by Eq. (1), with $J_{0}=\Delta_{0}=\lambda$ and $\alpha=\beta$. For this case,

$$
W=\frac{1}{2 \pi} \int_{-\pi}^{\pi} \frac{\left(\frac{A}{Q^{2}}+\frac{B}{Q}\right)}{\left(\frac{4 \lambda^{2} P^{2}}{Q}+1\right)} d k,
$$

where

$$
\begin{aligned}
& P=\left(2^{-\alpha} \sin (2 k)+3^{-\alpha} \sin (3 k)+\sin (k)\right), \\
& Q=\left(-2 \lambda\left(2^{-\alpha} \cos (2 k)+3^{-\alpha} \cos (3 k)+\cos (k)\right)-\mu\right), \\
& A=4 \lambda^{2} P\left(-2^{1-\alpha} \sin (2 k)-3^{1-\alpha} \sin (3 k)-\sin (k)\right), \\
& B=2 \lambda\left(2^{1-\alpha} \cos (2 k)+3^{1-\alpha} \cos (3 k)+\cos (k)\right) .
\end{aligned}
$$

Here the model should contain four topologically distinct phases, i.e., $W=0,1,2$, and 3 , respectively. However, we observe the suppression of $W=2$ region. For $\alpha=0,0.1$, there is transition among $W: 3 \leftrightarrow 0$ and $W: 1 \leftrightarrow 0$. For $\alpha \geqslant$ 0.3 , we see only $W=1$ and $W=0$ phases. When $\alpha=0$, the second and fourth critical lines superpose on each other 
(a)

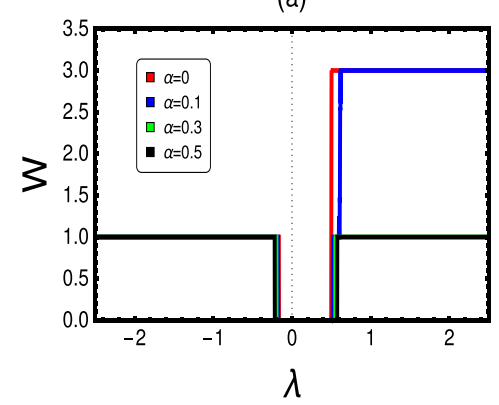

(b) $\lambda=1$

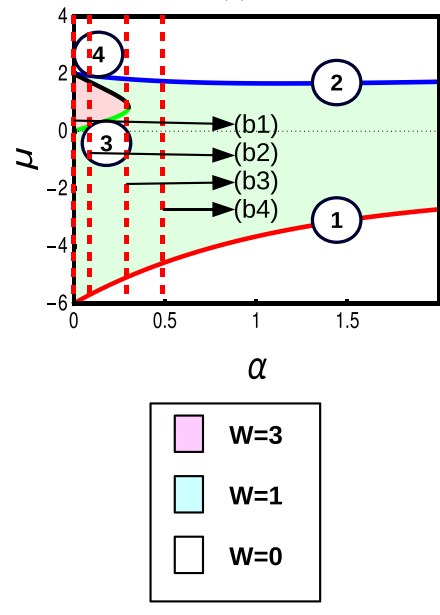

(b1) $\alpha=0$

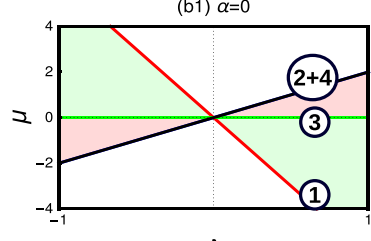

$\lambda$

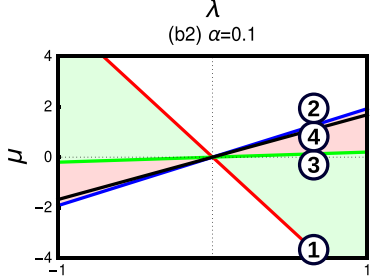

$\lambda$

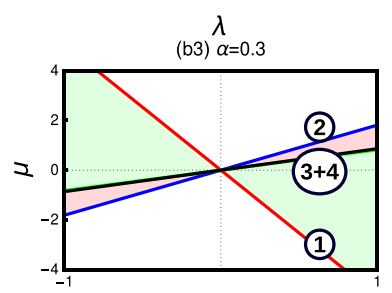

(b4) $\underset{\alpha=0.5}{\lambda}$

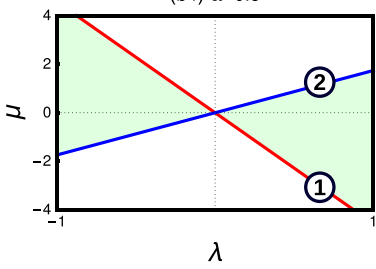

FIG. 2. Topological phase diagram of longer-range Kitaev chain with $r=3$ neighbors. (a) Winding number study with respect to $\lambda$ for different values of $\alpha$. (b) Behavior of topological phases in $\mu-\alpha$ parameter space with fixed $\lambda$. [(b1)-(b4)] Corresponding topological phase diagrams of model in $\mu-\lambda$ parameter space with increasing values of $\alpha$. This helps to understand the behavior of TQCLs. Red, blue, green, and black lines represent the first, second, third, and fourth TQCLs, respectively. [(b1) and (b2)] Initially the second and fourth TQCLs superpose on each other and gradually second TQCL shifts upward with increasing value of $\alpha$. [(b3) and (b4)] Third and fourth lines superpose on each other and vanish with the increasing value of $\alpha$.

and results in the suppression of $W=2$ phase. But when we gradually increase the value of $\alpha$, the third and fourth critical lines vanish as shown in Figs. 2(b1)-2(b4). So throughout this precess, $W=2$ region is absent. Hence the model transforms to original Kitaev model.

\section{Case 3: when $r=4$}

Figure 3(a) shows the phases for $r=4$. Here we can see the interaction is up to the fourth nearest neighbor in the chain and the gap closing occurs at five different values of $k$ (Appendix) which corresponds to five different TQCL. Technically the model should contain five topologically distinct phases, i.e., $W=0,1,2,3$, and 4, respectively. For this case, WN is given by

$$
W=\frac{1}{2 \pi} \int_{-\pi}^{\pi} \frac{\left(\frac{P}{B^{2}}+\frac{Q}{B}\right)}{\left(\frac{4 \lambda^{2} A^{2}}{B^{2}}+1\right)} d k
$$

where

$$
\begin{aligned}
& A=\left(\frac{\sin (2 k)}{2^{\alpha}}+\frac{\sin (3 k)}{3^{\alpha}}+\frac{\sin (4 k)}{4^{\alpha}}+\sin (k)\right), \\
& B=-2 \lambda\left(\frac{\cos (2 k)}{2^{\alpha}}+\frac{\cos (3 k)}{3^{\alpha}}+\frac{\cos (4 k)}{4^{\alpha}}+\cos (k)\right)-\mu, \\
& P=4 \lambda^{2} A\left(-\frac{\sin (2 k)}{2^{-1+\alpha}}-\frac{\sin (3 k)}{3^{-1+\alpha}}-\frac{\sin (4 k)}{4^{1-\alpha}}-\sin (k)\right), \\
& Q=2 \lambda\left(\frac{\cos (2 k)}{2^{-1+\alpha}}+\frac{\cos (3 k)}{3^{-1+\alpha}}+\frac{\cos (4 k)}{4^{-1+\alpha}}+\cos (k)\right) .
\end{aligned}
$$

When $\alpha=0$, we observe the transition among $W: 4 \leftrightarrow 0$ and $W: 1 \leftrightarrow 0$. When $\alpha=0,0.1$, there is a transition among $W: 0 \leftrightarrow 4$ and $4 \leftrightarrow 2$. When $\alpha=0.4,0.9$, there occurs a transition among $W: 2 \leftrightarrow 1,2 \leftrightarrow 0$ and $W: 1 \leftrightarrow 0$. When $\alpha=1,1.1$, there is a transition only among $W: 1 \leftrightarrow 0$, which represents original Kitaev chain. For $\alpha=0,0.1$, there is a superposition among third and fourth critical lines which results in the suppression of $W=3$ and $W=2$ phases. In the beginning, the second critical line lies on the $\lambda$ axis along with fifth critical line and gradually shifts upward with the increase of $\alpha$ [Figs. 3(b1)-3(b6)]. This results in the formation of $W=2$ region. The fourth critical line fails to distinguish the topological phases and the fifth critical line vanishes for $\alpha>0.1$, thus throughout the process $W=3$ phase is suppressed. Hence once again the model shifts to original Kitaev chain.

From this section, one can summarize the observations as following. (1) We notice that by increasing the number of interacting neighbors $r$ it is possible to get higher order WNs whereas if we increase the decay parameter $\alpha$ the higher order WNs vanish. (2) The reduction of higher order WNs to lower order occurs through the process of superposition and vanishing of TQCLs. (3) Higher order WNs are less stable (decay early) compared to its lower orders. In the same way, the TQCLs associated with higher order WN are also less stable and undergo superposition/vanishing early compared to its lower orders. (4) We also notice that it is not possible to achieve all the intermediate higher order WNs. This is because some of the TQCLs responsible for those particular intermediate WN undergoes superposition and results in the suppression of corresponding topological phases.

\section{CHARACTERISTIC STUDY OF CRITICAL LINES}

The conclusion drawn from the previous section leads to further analysis of the TQCLs as a function of $\alpha$. Our goal is uncovering the nature of the resultant TQCL when there exists multicriticality, i.e., when two TQCLs of distinct natures superpose on each other. Also we would like to analyze the stability of the different TQCLs as $\alpha$ varies. To understand these factors, we analyze the Berry connection and groundstate energy of the system in this section.

\section{A. Superposition of critical lines: an analysis of Berry connection}

Now we present the physical explanations of superposition of TQCLs from the perspective of curvature function. Curvature function of Bloch state is an important quantity whose 
(a) $\mu=1$

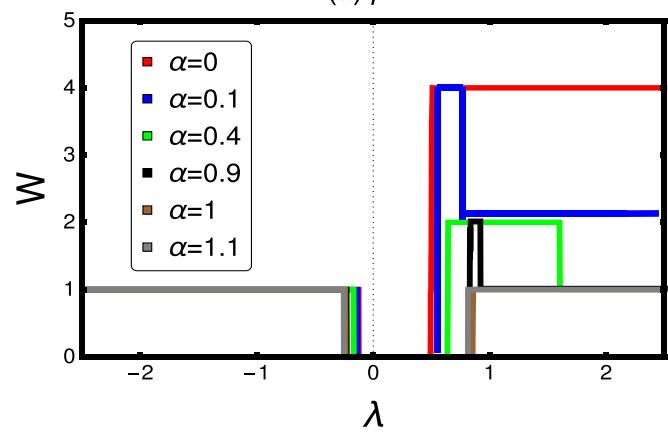

(b1) $\alpha=0$

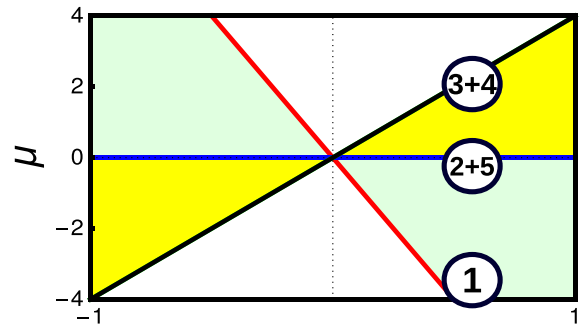

$\lambda$

(b4) $\alpha=0.9$

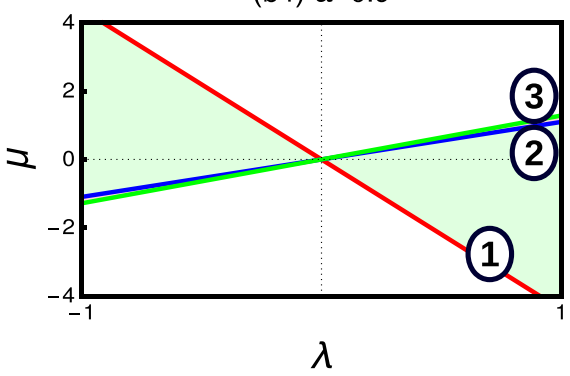

(b) $\lambda=1$
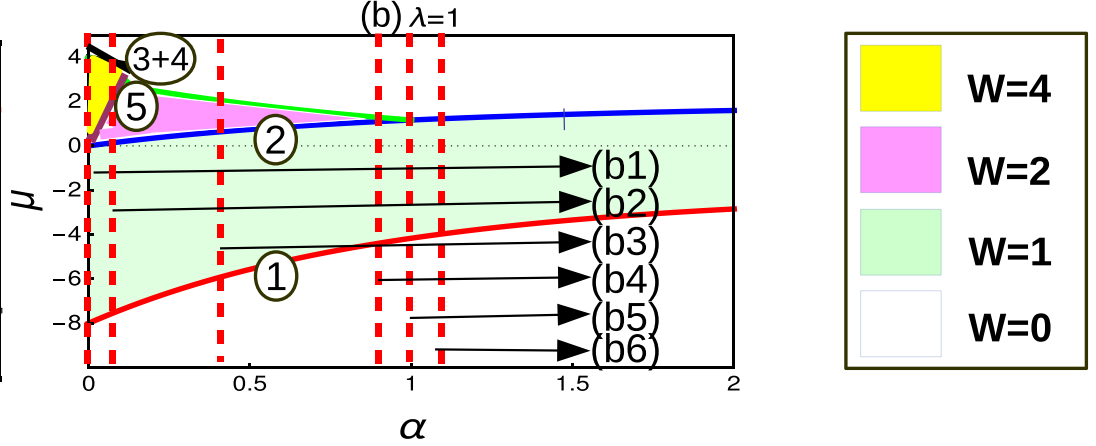

(b2) $\alpha=0.1$

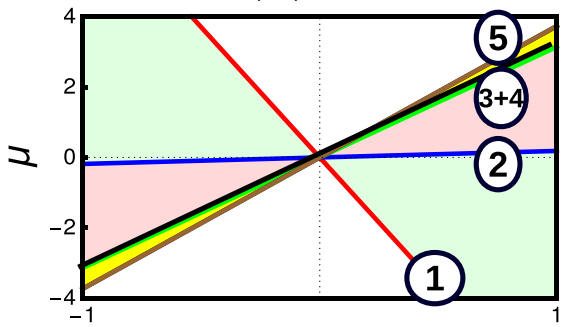

$\lambda$

(b5) $\alpha=1$

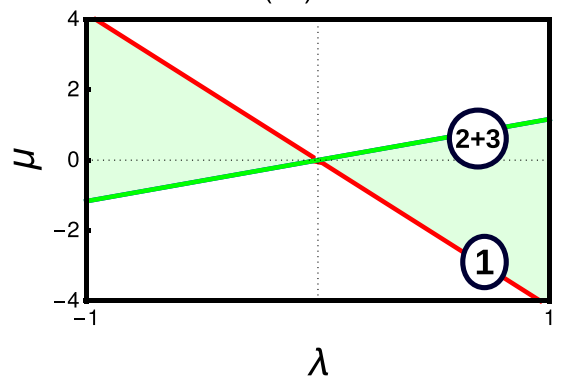

(b3) $\alpha=0.4$

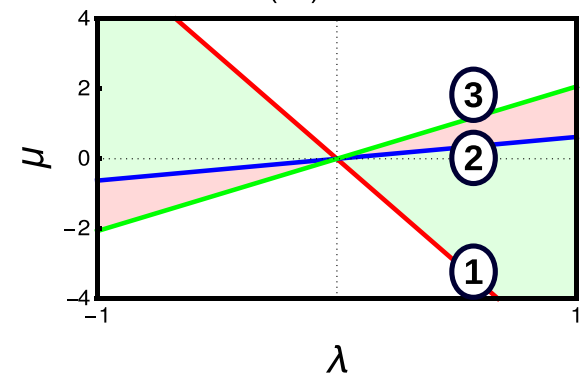

(b6) $\alpha=1.1$

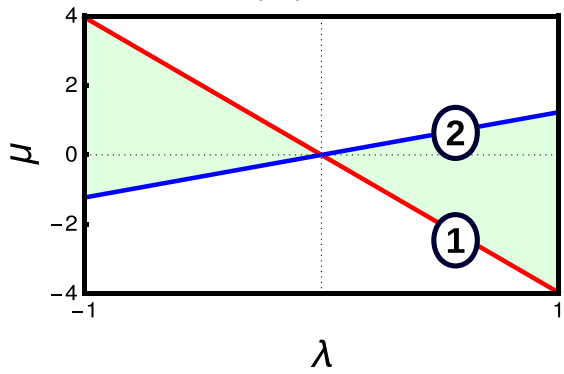

FIG. 3. Topological phase diagram of longer-range Kitaev chain with $r=4$ neighbors. (a) Winding number study with respect to $\lambda$ for different values of $\alpha$. (b) Behavior of topological phases in $\mu-\alpha$ parameter space with fixed $\lambda$. [(b1)-(b6)] Corresponding topological phase diagrams of model in $\mu-\lambda$ parameter space with increasing values of $\alpha$. This helps to understand the behavior of TQCLs. Red, blue, green, black and brown lines represent the first, second, third, fourth, and fifth TQCLs respectively. Initially the second TQCL lies on $\lambda$ axis superposed with and gradually moves upward with increasing $\alpha$. (b1) There is superposition among third-fourth and second-fifth TQCLs. (b2) Fifth TQCL shifts upward and third-fourth TQCLs remain as before. [(b3) and (b4)] With the increasing $\alpha$, fourth and fifth TQCLs vanish by yielding imaginary values. (b5) Third TQCL superposes with second TQCL. (b6) Third TQCL vanishes and model reduces to short-range Kitaev chain.

integral over the Brillouin zone defines the topological invariant $[16,50]$. The curvature function can take various forms like Berry connection, Berry curvature and Pfaffian of Bloch states. Here we consider Berry connection as our curvature function $F(k, M)$.

Berry connection (BC) is a momentum dependent function which diverges at specific points in the Brillouin zone as one approaches the critical values in the parameter space ( $\mathbf{M} \rightarrow \mathbf{M}_{\mathbf{c}}$, where $\mathbf{M}$ is the set of all parameters). If these points in the Brillouin zone have a symmetry $k_{0}=-k_{0}$, then they are called as the high symmetry points (HSP) [Figs. 4(a) and 4(b)]. Usually the BC behaves as an even function (i.e., $\left.F\left(k_{0}+\delta k, M\right)=F\left(k_{0}-\delta k, M\right)\right)$ around such points. There are also points in the Brillouin zone, where the symmetry $k_{0}=-k_{0}$ is not obeyed. These are non-HSPs which lies in the Brillouin zone other than $k=0$ and $k=\pi$. As the parameters approaches critical value $\mathbf{M} \rightarrow \mathbf{M}_{\mathbf{c}}$, the diverging peak of $\mathrm{BC}$ shifts towards non-HSP [Fig. 4(c)]. In both HSP, as well as non-HSP the BC diverges as one approaches the critical point $\left(\mathbf{M} \rightarrow \mathbf{M}_{\mathbf{c}}\right)$. As the critical point is crossed, the BC flips its sign, but point of divergence in $k$-space remains same. This is the generic nature of HSP. In the same way, even for a non-HSP, the BC tends to diverge as one approaches critical point. The point of divergence shifts based on the parameter space. This is the behavior of non-HSP.

Around all the critical points, the BC shows nonanalytic behavior and acquires the Ornstein-Zernike form around HSPs [16], i.e.,

$$
F\left(k_{0}+\delta k, \mathbf{M}\right)=\frac{F\left(k_{0}, \mathbf{M}\right)}{1+\xi_{k_{0}}^{2} \delta k^{2}} .
$$

When $k=k_{0}$ and with $\mathbf{M} \rightarrow \mathbf{M}_{\mathbf{c}}$ the length scale diverges $\left(\xi_{k_{0}} \rightarrow \infty\right)$, which results in the narrowing of Lorentzian term of Eq. (12). Thus one can observe the divergence of BC as $\mathbf{M}$ 
(a) $\operatorname{HSP}(\mathrm{k}=0), \lambda=0.5, \alpha=0$

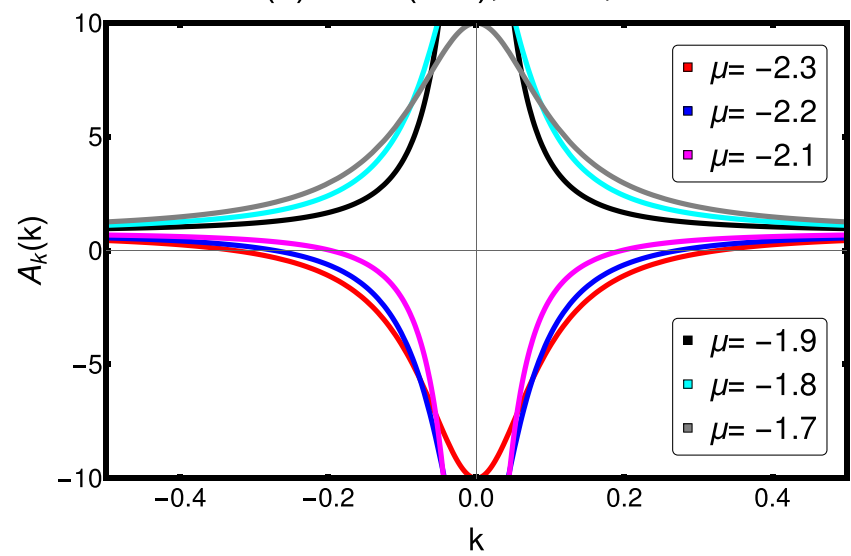

(b) $\operatorname{HSP}(\mathrm{k}=\pi), \lambda=1, \alpha=0$

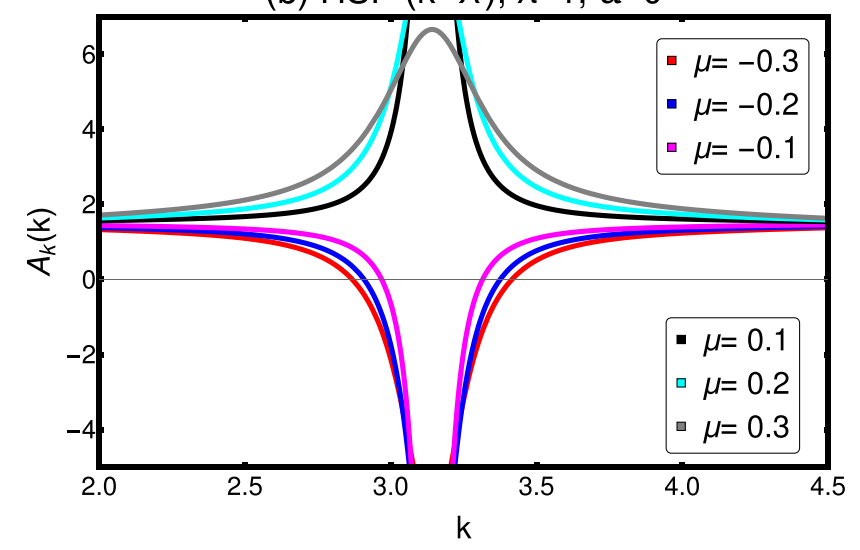

(c) Non-HSP $\left(k=\cos ^{-1}\left(-2^{\alpha-1}\right)\right), \lambda=1, \alpha=0$

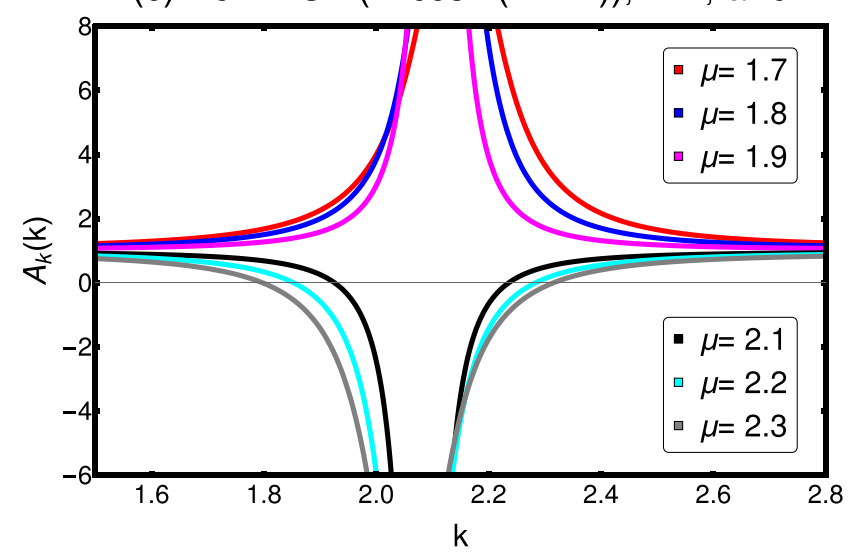

FIG. 4. Behavior of Berry connection for $r=2$ for (a) first TQCL with HSP at $k=0$ (b) second TQCL with a HSP at $k=\pi$, (c) third TQCL with a non-HSP $k=\cos ^{-1}\left(-2^{\alpha-1}\right)$.

is approached from both sides of critical line [16], i.e.,

$$
\lim _{\mathbf{M} \rightarrow \mathbf{M}_{c}^{+}} F\left(k_{0}, \mathbf{M}\right)=-\lim _{\mathbf{M} \rightarrow \mathbf{M}_{c}^{-}} F\left(k_{0}, \mathbf{M}\right)= \pm \infty .
$$

Close to the critical point, the $\mathrm{BC}$ follows the relation:

$$
F\left(k_{0}, \mathbf{M}\right) \propto\left|\mathbf{M}-\mathbf{M}_{c}\right|^{-\gamma} \text { and } \xi\left(k_{0}, \mathbf{M}\right) \propto\left|\mathbf{M}-\mathbf{M}_{c}\right|^{-v},
$$

where the exponents $\gamma$ and $v$ corresponds to the susceptibility and localization critical exponents, respectively. For a one- dimensional model, exponents obey the scaling law $\gamma=v$ and take modified forms near multicritical points [48].

Here we try to analyze the behavior of TQCLs with the decay parameter $\alpha$. We consider some cases for different values of $r$ and analyze the BC to understand the behavior of TQCLs when they undergo the process of superposition. The $\mathrm{BC}$ for a Bloch state across the Brillouin zone is defined as

$$
A_{k}=-2 i\left\langle u_{n k}\left|i \partial_{k}\right| u_{n k}\right\rangle=\frac{\chi_{z} \partial_{k} \chi_{y}-\chi_{y} \partial_{k} \chi_{z}}{\chi_{z}^{2}+\chi_{y}^{2}},
$$

where $\left|u_{n k}\right\rangle=\frac{1}{\sqrt{2} \chi}\left(\begin{array}{c}-\chi \\ \chi_{z}+i \chi_{y}\end{array}\right)$. For example, when $r=2$,

$$
A_{k}=\frac{2 \lambda\left(2\left(4^{\alpha}+2\right) \lambda+2^{\alpha} A\right)}{4 \lambda^{2}+4^{\alpha} B+2^{\alpha+2} \lambda(2 \lambda \cos (k)+\mu \cos (2 k))},
$$

where

$$
\begin{aligned}
& A=\cos (k)\left(2^{\alpha} \mu+6 \lambda\right)+2 \mu \cos (2 k), \\
& B=4 \lambda^{2}+4 \lambda \mu \cos (k)+\mu^{2} .
\end{aligned}
$$

Here HSPs are $k_{0}=0, \pi$ and non-HSP is $k_{0}=\cos ^{-1}\left(-2^{\alpha-1}\right)$. As there are just two interacting neighbors, we have three critical points (Appendix).

In a longer-range Kitaev model, there always exists two HSPs at $k=0$ and $k=\pi$. The number of non-HSPs depends on the number of interacting neighbors $r$ and higher order $r$ generates higher order TQCLs. When the decay parameter $\alpha$ starts to increase, the higher order TQCLs start to superpose on each other and vanish. Here we analyze different kind of superpositions: superposition of two HSP, superposition of HSP and non-HSP and superposition of two non-HSP. We study above combinations in the following.

\section{Case 1: when $r=2$}

Figure 5(a) shows the superposition of the second (HSP) and third (non-HSP) TQCL for $\alpha=1$ and $\lambda=1$. The second TQCL starts moving in the anticlockwise direction with increase in $\alpha$ (see Fig. 1). When $\alpha=0$ we can see the symmetric behavior of HSP at $k=\pi$. The $\mathrm{BC}$ tends to diverge at the point $(\mu=0, \alpha=0, \lambda=1)$ which happens to lie on the second TQCL [Fig. 4(b)]. As critical point $(\mu=2, \alpha=$ $0, \lambda=1$ ) on the third TQCL is crossed, the BC flips its sign, but the point of divergence in $k$ space is not same [Fig. 4(c)]. One can observe that the behavior of the plots in Fig. 5(a) is a combination of both HSP as well as non-HSP. As one approaches the critical point from the lower values of $\mu$ the $\mathrm{BC}$ behaves similar to that for a HSP i.e., it shows the peak around $k=\pi$ without any shift. But after the critical point $(\mu=1, \alpha=1, \lambda=1)$, it behaves as that for a non-HSP, i.e, the diverging peaks of $\mathrm{BC}$ starts shifting. This is an interesting phenomenon that occurs as a consequence of superposition of TQCLs.

\section{Case 2: when $r=3$}

In Fig. 5(b), we observe the superposition of second TQCL (HSP) and fourth TQCL (non-HSP) for $r=3$. Here we observe the symmetric nature of BC around the QCP $(\mu=$ $1, \alpha=0$, and $\lambda=0.5)$. As we approach the QCP from the lower values of $\alpha$, we observe the evenness of BC because of the high symmetric nature of second TQCL. As we pass 
(a) $r=2, \alpha=1, \lambda=1$

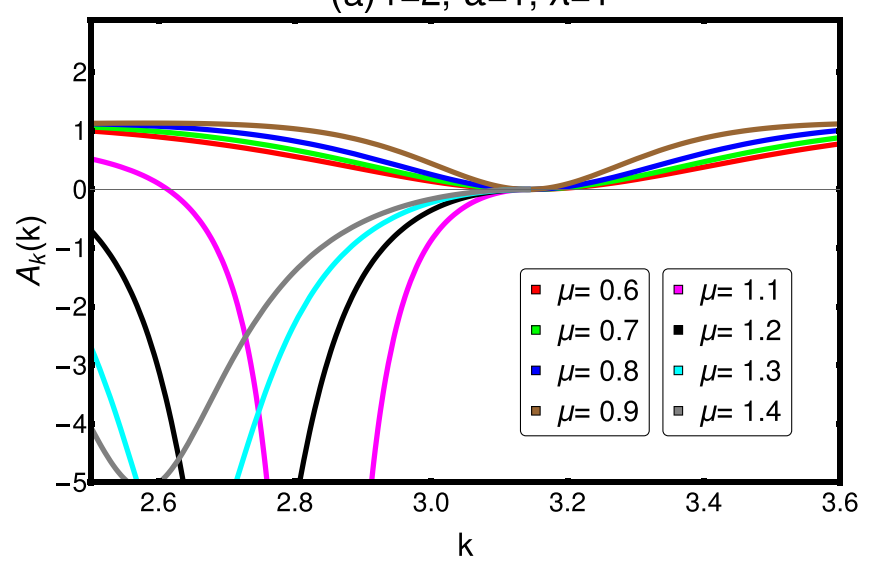

(b) $r=3, \alpha=0, \lambda=0.5$

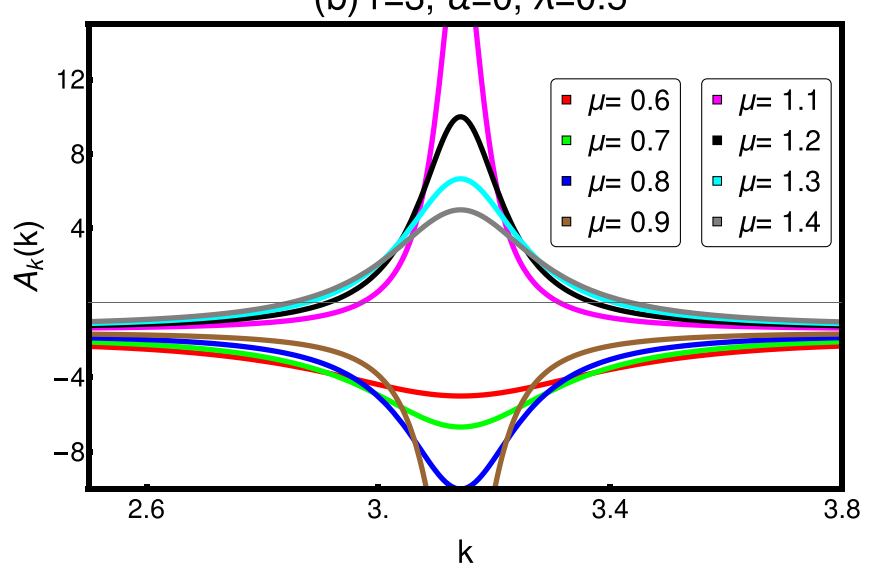

FIG. 5. Behavior of Berry connection for (a) $r=2$, superposition of second TQCL (HSP) and third TQCL (non-HSP) and (b) $r=$ 3 , superposition of second (HSP) and fourth TQCLs (non-HSP).

the QCP we observe the flip in the $\mathrm{BC}$, but still it exhibits high symmetric nature even though it is a non-HSP. We observe that the TQCLs associated with the higher order WNs are usually non-HSPs and are less stable with respect to $\alpha$. When they superpose with HSP, there occurs a very slight and insignificant shift in the diverging peaks, and hence they behave similar to HSP. In other way, the HSP dominates over the non-HSP, which is associated with higher order TQCLs.

\section{Case 3: when $r=4$}

In Fig. 6(a), we show the superposition second (HSP) and third (non-HSP) TQCLs for $r=4$. As one approaches QCP ( $\mu=0.58, \alpha=1$, and $\lambda=0.5$ ) from lower values of $\mu$ it exhibits the nature of HSP and as the QCP passes it exhibits the nature of non-HSP. Here we can observe the nature of both HSP as well as non-HSP. Fig. 6(b) shows the superposition of third and fourth TQCLs for $r=4$ where both of them are nonHSP. As one approaches QCP $(\mu=1, \alpha=0$, and $\lambda=0.5)$ from the lower values of $\mu$, the $\mathrm{BC}$ shows the non-HSP nature because of the effect of third TQCL. As the QCP is passed, the $\mathrm{BC}$ flips its sign, and still continue to behave as non-HSP. But we observe a comparatively less shift in the diverging peak of BC.

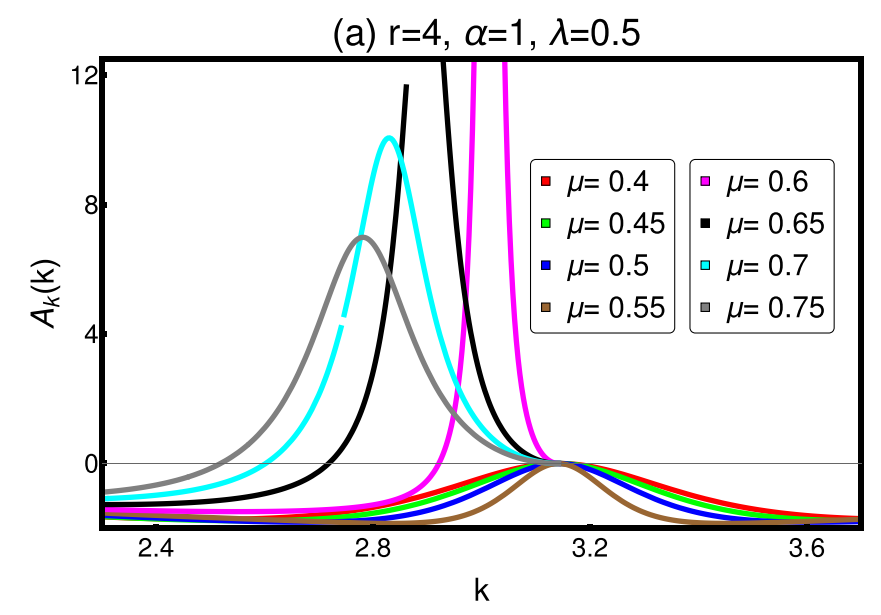

(b) $r=4, \alpha=0, \lambda=0.5$

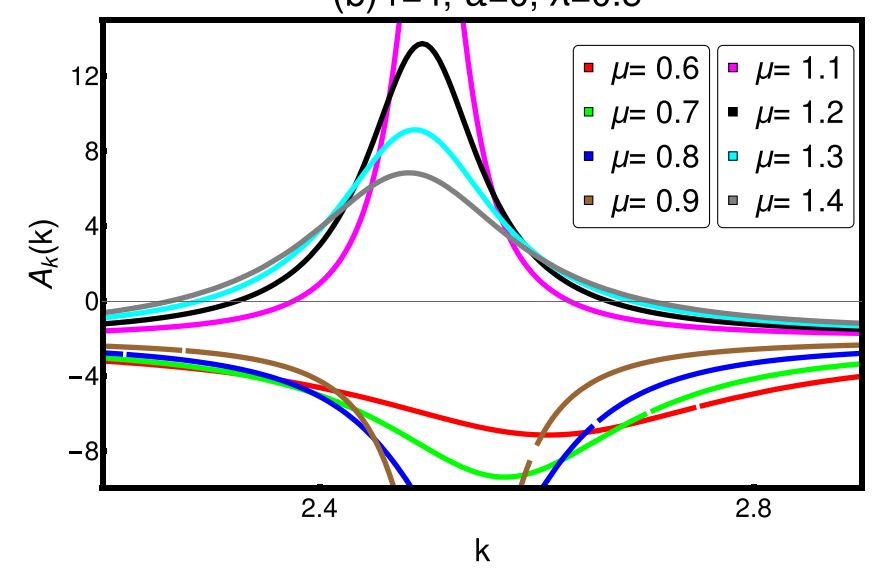

(c) $r=4, \alpha=0, \lambda=0.5$

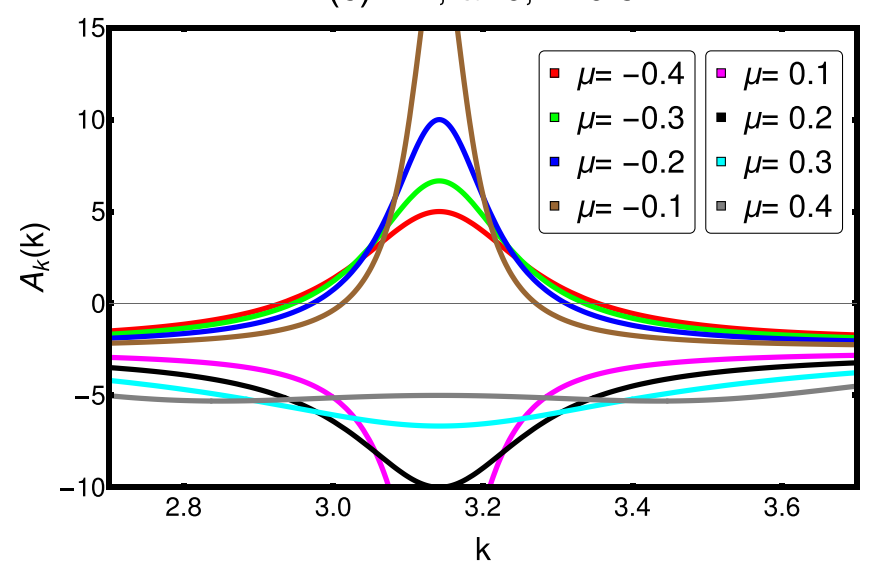

FIG. 6. Behavior of Berry connection for (a) $r=4$, superposition of second TQCL (HSP) and third TQCL (non-HSP); (b) $r=4$, superposition of third (non-HSP) and fourth (non-HSP) TQCLs; and (c) When $r=4$, superposition of second TQCL (HSP) and fifth TQCL (non-HSP).

In Fig. 6(c). we observe the superposition second (HSP) and fifth (non-HSP) TQCLs for $r=4$. As one approaches QCP $(\mu=0, \alpha=0, \lambda=0.5)$ from lower values of $\mu$ it exhibits the nature of HSP and as the QCP passes, even then it exhibits the nature similar to HSP. Here the non-HSP nature is very less expressive and one can observe an insignificant 
TABLE I. Possible parameter values of pseudospin vectors after series expansion. Here the $\delta g$ gives the criticality (M $\left.\rightarrow \mathbf{M}_{\mathbf{c}}\right)$ condition. The terms $A$ and $B$ decide the values of characteristic length critical exponent. In all the above cases, for $\alpha>1$, the term $A$ dominates and critical exponents yield $\gamma=v=1$. However, for $\alpha<1$, the values of $v$ and $\gamma$ varies depending on the number of interacting neighbors $r$.

\begin{tabular}{|c|c|c|c|c|}
\hline $\begin{array}{l}\text { Number } \\
\text { of } \\
\text { neighbors }\end{array}$ & $\begin{array}{l}\text { Critical } \\
\text { condition } \\
(\text { HSP })\end{array}$ & $A$ & $B$ & $\delta g$ \\
\hline \multirow[t]{2}{*}{$r=2$} & $k=0$ & $2 \lambda\left(1+\frac{2}{2^{\alpha}}\right)$ & $\lambda\left(1+\frac{4}{2^{\alpha}}\right)$ & $-\mu-2 \lambda\left(1+\frac{2}{2^{\alpha}}\right)$ \\
\hline & $k=\pi$ & $2 \lambda\left(-1+\frac{2}{2^{\alpha}}\right)$ & $\lambda\left(-1+\frac{4}{2^{\alpha}}\right)$ & $-\mu-2 \lambda\left(-1+\frac{2}{2^{\alpha}}\right)$ \\
\hline \multirow[t]{2}{*}{$r=3$} & $k=0$ & $2 \lambda\left(1+\frac{2}{2^{\alpha}}+\frac{3}{3^{\alpha}}\right)$ & $\lambda\left(1+\frac{4}{2^{\alpha}}+\frac{9}{3^{\alpha}}\right)$ & $-\mu-2 \lambda\left(1+\frac{2}{2^{\alpha}}+\frac{3}{3^{\alpha}}\right)$ \\
\hline & $k=\pi$ & $2 \lambda\left(-1+\frac{2}{2^{\alpha}}-\frac{3}{3^{\alpha}}\right)$ & $\lambda\left(-1+\frac{4}{2^{\alpha}}-\frac{9}{3^{\alpha}}\right)$ & $-\mu-2 \lambda\left(-1+\frac{2}{2^{\alpha}}-\frac{3}{3^{\alpha}}\right)$ \\
\hline \multirow[t]{2}{*}{$r=4$} & $k=0$ & $2 \lambda\left(1+\frac{2}{2^{\alpha}}+\frac{3}{3^{\alpha}}+\frac{4}{4^{\alpha}}\right)$ & $\lambda\left(1+\frac{4}{2^{\alpha}}+\frac{9}{3^{\alpha}}+\frac{16}{4^{\alpha}}\right)$ & $-\mu-2 \lambda\left(1+\frac{2}{2^{\alpha}}+\frac{3}{3^{\alpha}}+\frac{4}{4^{\alpha}}\right)$ \\
\hline & $k=\pi$ & $2 \lambda\left(-1+\frac{2}{2^{\alpha}}-\frac{3}{3^{\alpha}}+\frac{4}{4^{\alpha}}\right)$ & $\lambda\left(-1+\frac{4}{2^{\alpha}}-\frac{9}{3^{\alpha}}+\frac{16}{4^{\alpha}}\right)$ & $-\mu-2 \lambda\left(-1+\frac{2}{2^{\alpha}}\right.$ \\
\hline
\end{tabular}

shift of diverging BC peaks. Hence the HSP dominates over non-HSP of higher order TQCLs similar to Fig. 5(b).

The first TQCL (HSP $k=0$ ) does not involve in any superposition phenomena with the increasing values of $\alpha$. For a short-range model, the region bounded between two HSP $(k=0, \pi)$ gives $W=1$ topological phase which is also the characterizing nature of Kitaev chain. As $\alpha \rightarrow \infty$ all the longer-range models reduce to original Kitaev chain. Hence there is no chance for superposition of two $\operatorname{HSP}(k=0, \pi)$. If such case occurs, then there will be an absence of $W=1$ phase.

\section{Critical exponents for longer-range models}

Critical exponents are the quantities which explain the behavior of the system around the criticality. However, the TQPTs are second-order phase transitions where one can calculate the critical exponents by expanding the pseudospin vectors around at $k=k_{0}$ as

$$
\left.\chi(k)\right|_{k=k_{0}}=\chi\left(k_{0}\right)+\chi^{\prime}\left(k_{0}\right) k+\chi^{\prime \prime}\left(k_{0}\right) k^{2} / 2 .
$$

By substituting the expanded form of pseudospin vectors in Eq. (15), which leads to the Ornstein-Zernike form $[12,48]$, i.e.,

$$
\begin{aligned}
\left.F(k, \mathbf{M})\right|_{k=k_{0}} & =\frac{A \cdot \delta k(2 B . \delta k)-\left(\delta g+B \delta k^{2}\right) A}{\delta g^{2}+\left(2 B \delta g+A^{2}\right) \delta k^{2}+B^{2} \delta k^{4}} \\
& =\frac{\frac{2 A B \delta k^{2}-A\left(\delta g+B \delta k^{2}\right)}{\delta g^{2}}}{1+\left(\frac{2 \delta g \cdot B+A^{2}}{\delta g^{2}}\right) \delta k^{2}+\left(\frac{B^{2}}{\delta g^{2}}\right) \delta k^{4}} \\
& =\frac{F\left(k_{0}, \delta g\right)}{1+\xi^{2} \delta k^{2}+\xi^{4} \delta k^{4}},
\end{aligned}
$$

where $\xi$ is the characteristic length. The terms $A, B$ and $\delta g$ are the parameters that come from Taylor series of expansion. In Eq. (18), there are two terms which decides the characteristic length. 1) $\xi \propto \sqrt{\frac{2 B}{\delta g}+\frac{A^{2}}{\delta g^{2}}}$, where the term $\frac{A^{2}}{\delta g^{2}}$ dominates over $\frac{2 B}{\delta g}$. Hence $\xi \propto 1 /|\delta g| \Rightarrow v=1$. 2) $\xi \propto$ $\sqrt[4]{\frac{B^{2}}{\delta g^{2}}}$ and $\xi \propto|\delta g|^{-1 / 2} \Rightarrow v=1 / 2$. Thus the dominating term among $A$ and $\sqrt{B}$ decides the characteristic length critical exponent. The exponent of the numerator gives the susceptibility critical exponent [48]. In Table I, we calculate the terms $A, B$ and $\delta g$ of a longer-range Kitaev chain with different number of interacting neighbors $r$. These critical exponents only correspond to HSPs, which always fit into scaling law whenever they are away from multicritical points as well as superposition of TQCLs. In a one-dimensional system, the critical exponents of Eq. (14) follow the scaling rule $v=\gamma$ [12]. However, when there exists some multicriticality or superposition of critical lines, this relation does not hold good [48]. This is because of the unevenness of the BC around those critical point. Around the superposition of TQCL one can observe the violation of even nature of BC throughout the BZ (i.e., $\lim _{\mathbf{M} \rightarrow \mathbf{M}_{c}^{+}} F\left(k_{0}, \mathbf{M}^{+}\right) \neq$ $\left.-\lim _{\mathbf{M} \rightarrow \mathbf{M}_{c}^{-}} F\left(k_{0}, \mathbf{M}^{-}\right)= \pm \infty\right)[16]$. For a non-HSP, the curvature function fails to acquire the Ornstein-Zernike form, and thus it is not possible to do conventional scaling in such cases [51].

The scaling scheme also fails at high-symmetry points where the BC attains the fixed point configuration [48]. This kind of behavior can be observed in Fig. 5(a) and 6(a). The fixed point configuration of the $\mathrm{BC}$ is the state where the height of the curve does not vary along with the varying parameter, which can occur as a consequence of the multicritical point. Hence it is not possible to calculate the critical exponents $v$ and $\gamma$ at these points.

Through the study of BC we notice following points.

(1) Longer-range models contains a number of symmetry points (HSP/non-HSP) depending on the number of interacting neighbors. (2) As the decay parameter $\alpha \rightarrow \infty$, the longer-range model reduces to short-range where only HSP remains. (3) When two TQCLs superpose on each other, both TQCLs influence each other. Because of this reason, we can find mixed nature in the resultant TQCL. (4) The points corresponding to $k=0$ and $k=\pi$ represents HSP and all other symmetry points correspond to non-HSP. The non-HSPs corresponding to higher order WN or higher TQCL are comparatively less expressive in their nature. (5) The lower order symmetry points dominate over the higher order symmetry points when they superpose on each other. (6) It is not possible to find the critical exponents near the multicritical points as well as at superposition of TQCLs.

\section{B. Vanishing of critical lines}

TQCLs are the boundaries which separates distinct topological phases and manifests as a gap closing in the quasienergy spectrum. All the TQCLs lead to the gap closing 
but all the gap closings need not correspond to TQCLs. Vanishing of TQCL occurs through different means as follows: (1) by creating the gap openings in the quasienergy spectrum. (2) The critical line acquires complex values and at some point real part vanishes and only imaginary part remains (complex critical lines do not have any physical meaning thus we consider only real part of such critical lines).

TQPTs are the discontinuities in the second-order derivative of the ground-state energy of the system $[21,33,34]$. These discontinuities represent the nonanalyticities of the groundstate energy [28,52], i.e., the ground-state energy before and after TQPTs belongs to two different topological indices. This creates a discontinuity in the second-order derivative of the ground-state energy. In this section, we study ground-state energy of the system to understand the TQPT and vanishing of TQCL as mentioned earlier.

\section{Case 1: when $r=2$}

From Fig. 1, we observe that with the increasing values of $\alpha$, the $W=2$ phase vanishes along with its associated TQCL. To verify this we consider the third TQCL and study the quasienergy dispersion as a function of $\alpha$. One can always find a gapless point at $k=\cos ^{-1}\left(-2^{\alpha-1}\right)$ in the quasienergy spectrum for $\alpha<1$. However, the quasienergy spectrum becomes gapped $\forall k$ for $\alpha>1$ as shown in Fig. 7(a).

Another way to verify the vanishing of TQCL is through the study of ground-state energy of the Hamiltonian. In topological systems, a phase transition can be understood from the nonanalyticities of the ground-state energy [28,52]. Here we calculate the second-order derivative of ground-state energy $E^{\prime \prime}(\mu)$, given by

$$
\begin{aligned}
\frac{\partial^{2} E(\mu)}{\partial \mu^{2}} & =-\frac{1}{2 \pi} \int_{-\pi}^{\pi} \frac{\partial^{2}}{\partial \mu^{2}}\left(\sqrt{\left(\chi_{z}\right)^{2}+\left(\chi_{y}\right)^{2}}\right) d k, \\
& =-\frac{1}{2 \pi} \int_{-\pi}^{\pi} \frac{2 \lambda^{2} \sin ^{2}(k)\left(2^{\alpha}+2 \cos (k)\right)^{2}}{\left(4 \lambda^{2}+4^{\alpha} Q+2^{\alpha+2} \lambda A\right) \sqrt{P}} d k,
\end{aligned}
$$

where

$$
\begin{aligned}
& P=4^{1-\alpha} \lambda\left(4^{\alpha}(\mu \cos (k)+\lambda)+2^{\alpha} A+\lambda\right)+\mu^{2}, \\
& Q=4 \lambda^{2}+4 \lambda \mu \cos (k)+\mu^{2}, \\
& A=(2 \lambda \cos (k)+\mu \cos (2 k)) .
\end{aligned}
$$

Figure 7(b) shows the discontinuities (spikes) in $E^{\prime \prime}(\mu)$ for $\lambda=0.5, \alpha=1.5$, and for two different values of $\mu$, which correspond to the first and second TQCLs, respectively. We do not observe the third spike which is the signature for third TQCL. This means for $\alpha>1$, the third TQCL vanishes (see Appendix). When one increases the number of interacting neighbors $r$, the number of different topological phases increases leading to more number of TQCLs. As decay parameter $\alpha \rightarrow \infty$, gradually all higher order WNs along with TQCLs (corresponding to non-HSPs) vanish. At the end, only the TQCL which corresponds to $\operatorname{HSP}(k=0, \pi)$ remains. This remaining TQCL characterizes the TQPT between $W=$ 0 and $W=1$ phases in the original Kitaev chain. One can verify this by the quasienergy spectrum for $\alpha>1$. In the inset of Fig. 7(b), we study all three TQCL for $\alpha=1.5(\alpha>1)$. Here we can observe the gap closings only for $k=0$ and $\pi$, which correspond to the first and second TQCLs, respectively.

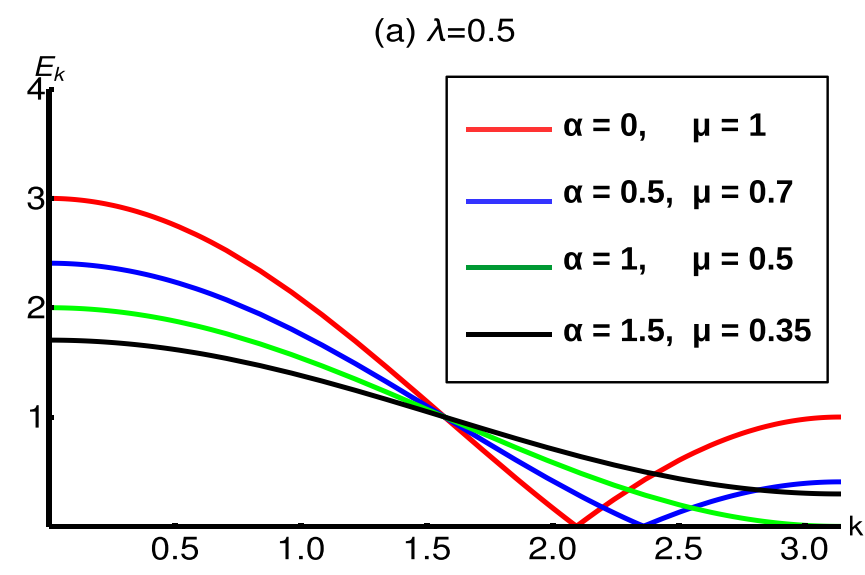

(b) $\alpha=1.5, \lambda=0.5$

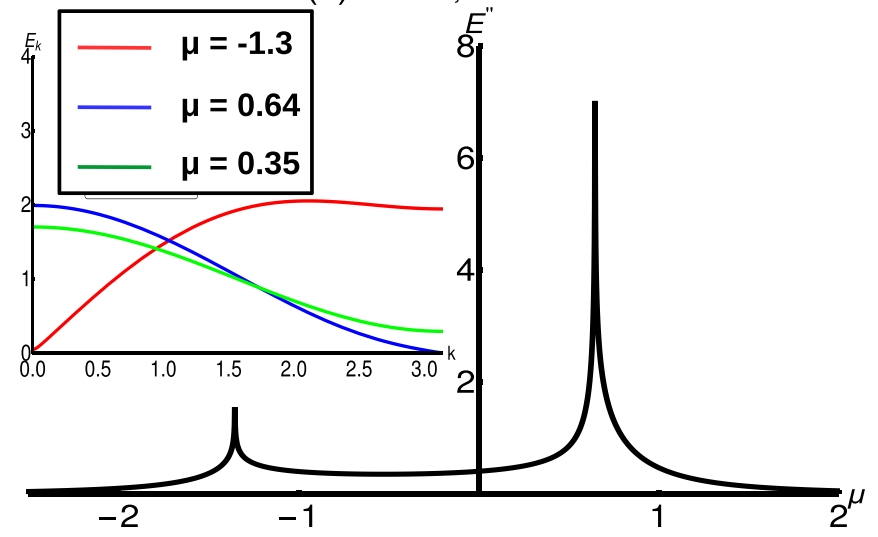

FIG. 7. The panel indicates the process of vanishing of critical line for $r=2$. (a) Gap closing of third critical line for different values of $\alpha$. Here the black line does not create gapless condition. (b) Discontinuity in the second-order derivative of ground-state energy. Two spikes corresponds to first and second TQCL respectively. (Inset) Behavior of all the three critical lines for $\alpha=1.5$. The green line (third TQCL) does not create gap closing.

There does not exist a third gap closing point. Hence it signals the absence of the third TQCL for $\alpha>1$.

\section{Case 2: when $r=3$}

Figure 8 represents the process of vanishing of TQCL for $r=3$. The system consists of four TQCLs among which the first two are HSP and later two are non-HSP, respectively. Initially, at $\alpha=0$, the energy spectrum corresponding to third TQCL is gapless and becomes gapped after $\alpha=0.2$ [Fig. 8(a)]. In the same way, the fourth TQCL also exhibits a transformation from gapless to gapped spectrum [Fig. 8(b)]. In both the cases, the gapped energy spectrum represents the vanishing of TQCL. At the end of process one can observe only two gapless TQCLs which correspond to HSP i.e., $k=0$ and $\pi$, respectively [Fig. 8(c)].

This can also be verified by the second-order derivative of the ground-state energy, given by

$$
\frac{\partial^{2} E(\mu)}{\partial \mu^{2}}=-\frac{1}{2 \pi} \int_{-\pi}^{\pi} \frac{2 \lambda^{2} P^{2}}{\pi\left(4 \lambda^{2} P^{2}+(\mu+2 \lambda Q)^{2}\right)^{3 / 2}} d k,
$$


(a) $\lambda=0.5$

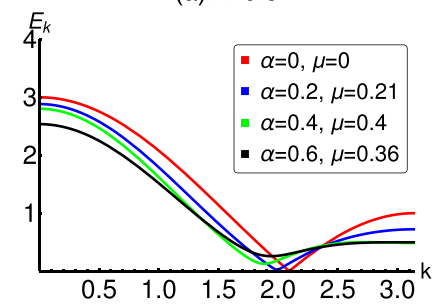

(c) $\alpha=0.6, \lambda=0.5$
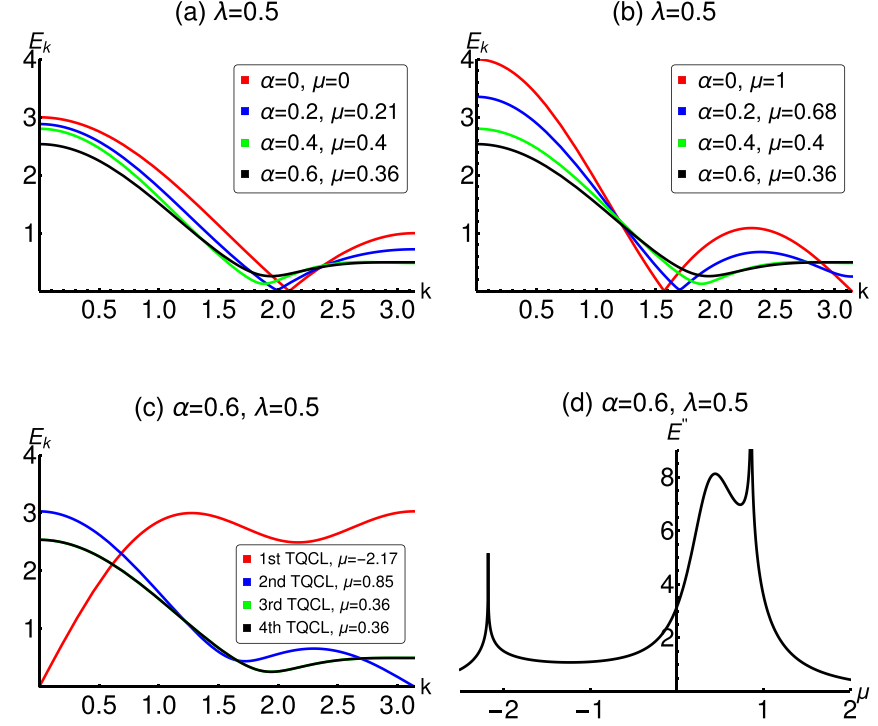

(d) $\alpha=0.6, \lambda=0.5$

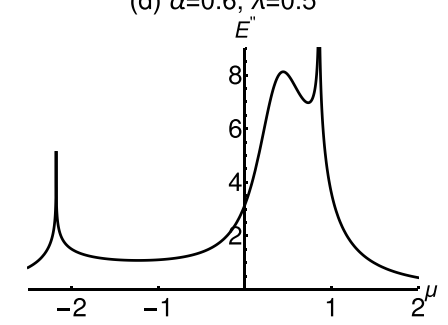

FIG. 8. The panel indicates the process of vanishing of critical line $r=3$. (a) Gap closing of the third TQCL for different values of $\alpha$. Here the green and black lines does not create gapless condition. (b) Gap closing of the fourth TQCL for different values of $\alpha$. Even here, the green and black lines does not create gapless condition. (c) Behavior of all the four TQCLs for $\alpha=0.6$. The green and black lines does not create gap closing. (d) Discontinuity in the secondorder derivative of ground-state energy. Two spikes corresponds to the first and second TQCL, respectively. There is no spike for the third and fourth TQCL.

where

$$
\begin{aligned}
& P=2^{-\alpha} \sin (2 k)+3^{-\alpha} \sin (3 k)+\sin (k), \\
& Q=2^{-\alpha} \cos (2 k)+3^{-\alpha} \cos (3 k)+\cos (k) .
\end{aligned}
$$

For $r=3$, the term $E^{\prime \prime}(k)$ shows only two nonanalyticities for $\alpha>0.2$ which correspond to HSPs [Fig. 8(d)]. The small hump before the second spike represents former QCP. With the increasing value of $\alpha$, the discontinuities in $E^{\prime \prime}$ (which correspond to third and fourth TQCL) vanish and gradually become a continuous curve. This signals the vanishing of third and fourth TQCL for higher values of $\alpha$.

\section{Case 3: when $r=4$}

As one goes for more number of interacting neighbors $r$, it is possible to witness complex TQCLs (see Appendix for details). As per present knowledge, complex TQCLs does not have any particular physical significance. In $r=4$ case, initially for lower values of $\alpha$ the 3rd, fourth and 5th TQCL participates in the process of superposition of TQCLs. But as $\alpha$ increases, the TQCLs becomes complex and gradually the real part becomes zero. The imaginary part does not contribute to the phase boundary of topological phases. Hence one can come to conclusion that, the occurrence of imaginary value is the signature of vanishing of TQCLs.
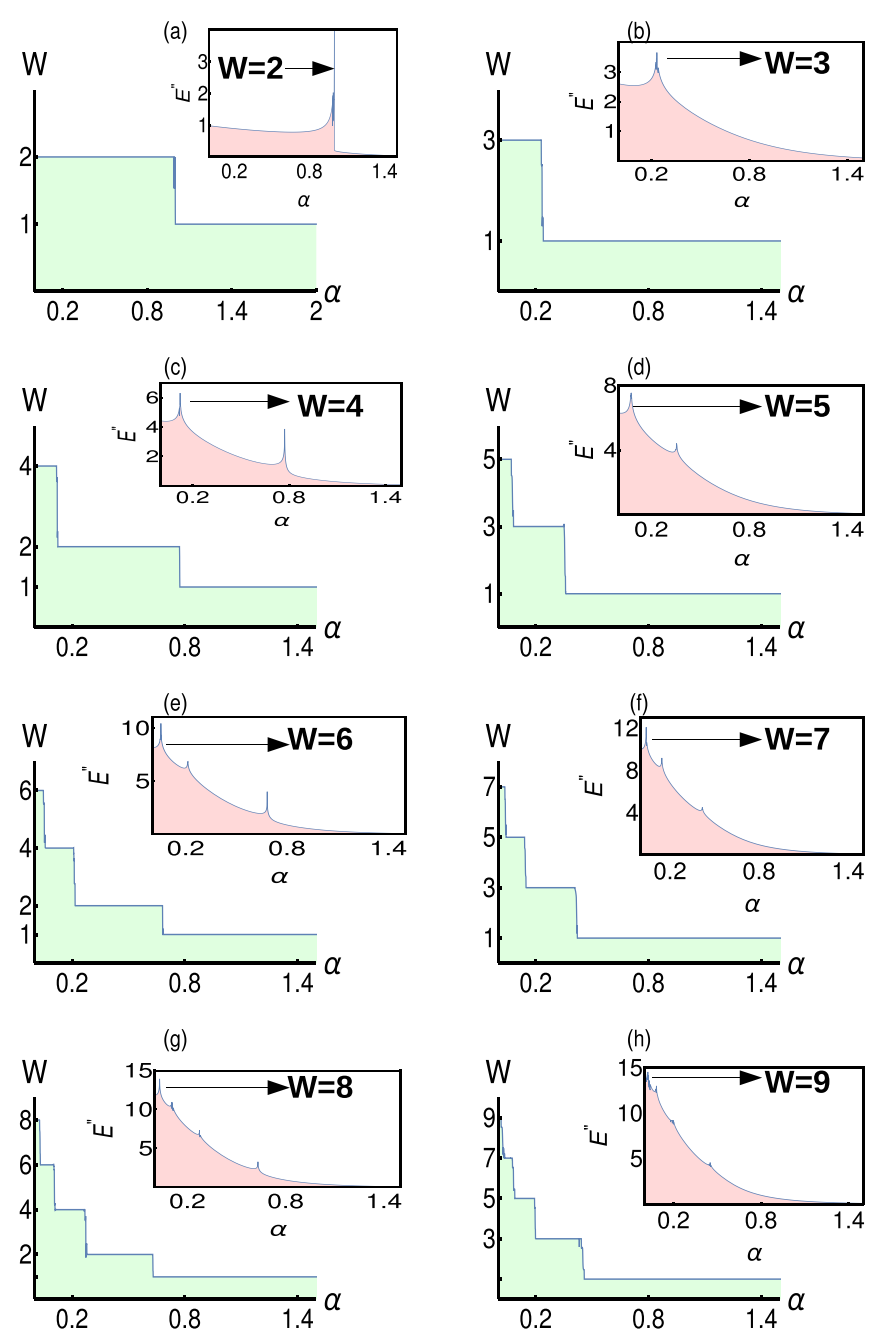

FIG. 9. Variation of winding number with $\alpha$ for the increasing numbers of interacting nearest neighbors $r=2,3,4, \ldots, 9$ as shown in figure (a), (b), (c),.., (h), respectively. The TQPT from higher WN to lower occurs among even-even or odd-odd WNs only. (Inset) The corresponding second-order derivative of the groundstate energy $\left(E "=\frac{\partial^{2} E}{\partial \alpha^{2}}\right)$ as a function of $\alpha$. The peaks in the plots denote the points of TQPTs. For all the plots we keep $\lambda=1$ and $\mu=1$.

\section{Longer-range effect and the stability of higher order winding numbers}

From Figs. 1-3, we understand certain behavior of the isotropic longer-range Kitaev chain. Here we study the fate of the topological phase with highest WN corresponding to a specific value of $r$ as the decay parameter $\alpha$ is varied from 0 to higher values. For better understanding, we consider number of interacting neighbors $r>4$ as shown in Fig. 9. We observe an interesting behavior of TQPT for the values $r>2$. There exist TQPTs from even-to-even and odd-to-odd WNs only for even and odd values of $r$, respectively.

It is well known that there exists a one-to-one correspondence between the WN as well as the localized edge modes of the system $[28,40]$. For a system with $r$ edge modes, there always exists different penetration lengths corresponding to each edge mode [11]. At TQPT this penetration length di- 
verges and edge mode merges with the bulk. For a system with higher $\mathrm{WN}$, due to the longer-range effect as one increases $\alpha$ the edge mode decays in a faster way. This creates an instability in such a way that the higher order $\mathrm{WN}$ reduces to its corresponding lower order. However, in the large $\alpha$ (shortrange) regime all higher order WNs $(W>1)$, irrespective of even or odd $W$, reduce to $W=1$ similar to the topological phase in the original Kitaev chain.

From the previous section, we observe different possibilities of superposition of TQCLs. Higher order of neighboring interaction generates higher order $\mathrm{WN}$ as well as corresponding higher order TQCLs. The higher order TQCLs are less stable with respect to $\alpha$ and continuously undergo superposition with its lower orders. These higher order TQCLs are comparatively less expressive in their nature and gets dominated by their lower order TQCLs when they undergo superposition. In the meantime, some of the higher order TQCL vanish either by creating a gap opening in the energy spectrum or by becoming complex. This results in the suppression of higher order WNs and the system reduces continuously to its lower order and finally to short-range Kitaev chain.

The inset of of Fig. 9 shows the second derivative of the ground-state energy

$$
E^{\prime \prime}(\alpha)=-\frac{1}{2 \pi} \int_{-\pi}^{\pi} \frac{\partial^{2} E}{\partial \alpha^{2}} d k,
$$

with $E$ being the ground-state energy, as a function of $\alpha$ (For a many-body system $E=-\sum_{k} E_{k}[28,52]$ and the summation is replaced by the integration when it comes to the Brillouin zone limit). The discontinuity in the derivative of the ground-state energy symbolizes the order of the quantum phase transition $[21,33,34]$. For a topological system, these discontinuities represent the nonanalyticities of the groundstate energy $[28,52]$. Here we observe discontinuity in second order derivative of energy with respect to $\alpha$, which indicates the transitions are second-order TQPTs. The maximum among the peaks signifies the TQPT from highest WN to consecutive even/odd WNs depending on even/odd $r$. For the higher order WN, the maximum peak shifts towards the lower values of $\alpha$ implying the shorter existence of these phases as $\alpha$ increases. We also notice that the amplitudes of peaks shift to higher values of $E^{\prime \prime}$ with increasing number of the interacting neighbors $r$.

\section{Analysis of long-range model through momentum space characterization}

In previous sections, we have studied the criticality and momentum space characterization of longer-range Kitaev chain with different number of neighbors. In this section, we consider Kitaev chain with infinite number of neighbors (i.e., $r \rightarrow \infty$ ) where the hopping term and the pairing terms decay with parameter $\alpha$ [Eq. (5)]. The corresponding energy dispersion is given by Eq. (6). Here both sine and cosine functions lead to polylogarithmic functions, given by

$$
\begin{aligned}
& \chi_{y}(k)=2 \lambda\left(\frac{L i_{\alpha}\left[e^{i k}\right]-L i_{\alpha}\left[e^{-i k}\right]}{2 i}\right), \\
& \chi_{z}(k)=-\mu-2 \lambda\left(\frac{L i_{\alpha}\left[e^{i k}\right]+L i_{\alpha}\left[e^{-i k}\right]}{2}\right),
\end{aligned}
$$

TABLE II. Possible TQCLs for different regimes of an isotropic long-range Kitaev chain. When $\alpha<1$, the TQCL does not exists as $k \rightarrow 0$.

\begin{tabular}{lcc}
\hline \hline Decay parameter & $k=0$ & $k=\pi$ \\
\hline When $\alpha>1$ & $\mu=-2 J_{0} \zeta[\alpha]$ & $\mu=-2 J_{0}\left(2^{1-\alpha}-1\right) \zeta[\alpha]$ \\
When $\alpha<1$ & - & $\mu=-2 J_{0}\left(2^{1-\alpha}-1\right) \zeta[\alpha]$ \\
\hline \hline
\end{tabular}

where we consider an isotopic long-range Kitaev chain for which $J_{0}=\Delta_{0}=\lambda$ and $\alpha=\beta$. The term $\chi_{y}$ [Eq. (20)] decides the number of gap closing points in the BZ and $\chi_{z}$ decides the parameters to be tuned for the critical conditions i.e., $\mathbf{M} \rightarrow \mathbf{M}_{c}$. The term $\Delta_{0}=\lambda$ in the $\chi_{y}$ represents the finite superconducting gap and does not influence the gap closing conditions of the system. The parameters $\mu$ and $J_{0}=\lambda$ act as the tuning parameters $(\mathbf{M})$ and $\chi_{z}$ gives the critical line which is nothing but the topological phase boundary. These critical lines assure the gap closing conditions during the topological phase transitions. When $\mathbf{M}$ is away from $\mathbf{M}_{c}$, the system is gapped and with $\mathbf{M} \rightarrow \mathbf{M}_{c}$ the system attains gapless condition.

In Eq. (20), pseudospin parameters exhibit polylogarithmic nature, where the gap closing $\left(\chi_{y} \rightarrow 0\right)$ occurs at $k=0$ and $k=\pi$ for different regime of decay parameter as shown in Table II and corresponding phase diagram is given by Fig. 10(a). When $k=\pi$, criticality occurs $\left(\mathbf{M} \rightarrow \mathbf{M}_{\mathbf{c}}\right)$, i.e., $E_{k} \rightarrow 0$ at $\mu=-2 J_{0}\left(2^{1-\alpha}-1\right) \zeta[\alpha]$ as $\chi_{y} \rightarrow 0$ for all values of $\alpha$. When $k=0$, criticality occurs at $\mu=-2 J_{0} L i_{\alpha}[1]$, where $\chi_{y} \rightarrow 0$ only for $\alpha>1$. Hence both $\alpha \leqslant 1$ and $\alpha>1$ belong to two different topological phases without a boundary. Figure 10(b) shows the energy spectrum of a long-range model, where $\alpha<$ 1 and $\alpha>1$, belonging to two different gapped phases with distinct topological properties, where the transition among (a)

(c)

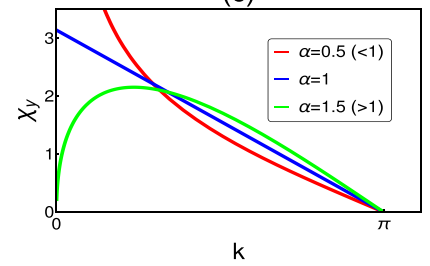

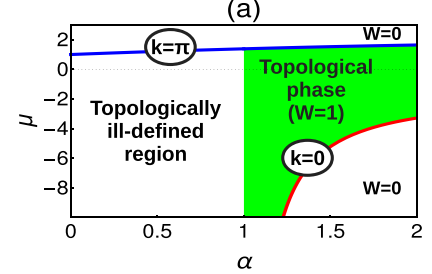

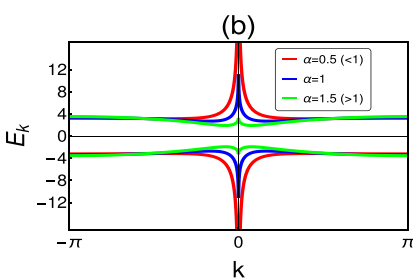

(d)

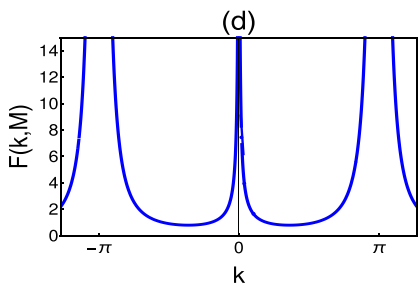

FIG. 10. (a) General phase diagram of isotropic long-range Kitaev chain with $\lambda=1$. (b) Energy dispersion for different values of $\alpha$. The system remains gapped for all values of $\alpha$ and $E_{k}$ diverges for $\alpha<1$. Here $\mu=-2, \lambda=1$, and system size $l=2000$. (c) Dispersion of the $\chi_{y}$ parameter at $k=0$ and $k=\pi$ for different values of $\alpha$ with $\mu=0, \lambda=1$ and system size $l=2000$. The term $\chi_{y}$ diverges, $k \rightarrow 0$ as $(k)^{\alpha-1}$ for all $\alpha<1$ and converges for $\alpha>1$. (d) Berry connection plot for $\alpha<1$, which shows the nonanalytic behavior through out the $\alpha<1$ region where $\mu=0$ and $\lambda=1$. 
them occurs at $\alpha=1$ without gap closing. In a similar model, transition without gap closing has been reported in Ref. [23]. This kind of bifurcation occurs due to the behavior of $\chi_{y}$ with respect to $\alpha \cdot \chi_{y}$ forms gapless condition at $k=\pi$ irrespective of $\alpha$ but it diverges and fails to make gapless condition at $k=0$ when $\alpha \leqslant 1$ [Fig. 10(c)]. However, due to this nature of $\chi_{y}$, Eq. (15) $\left[d \theta_{k} / d k=d\left(\tan ^{-1}\left(\chi_{y} / \chi_{z}\right)\right) / d k\right]$ also becomes nonanalytic for $\alpha \leqslant 1$ region even for gapped phases [Fig, $10(d)]$. Hence it is not possible to define integer $\mathrm{WN}$ for $\alpha \leqslant 1$, where $W$ takes positive and negative fractional values below and above the critical line $\mu=-2 J_{0}\left(2^{1-\alpha}-1\right) \zeta[\alpha]$, respectively.

This situation can be analyzed by expanding the polylogarithmic function around gap closing points. Expansions of polylogarithmic functions is given by [23,53],

$$
L i_{\alpha}\left[e^{i k}\right]=\Gamma[1-\alpha](-i k)^{\alpha-1}+\sum_{n=0}^{\infty} \frac{\zeta[\alpha-n]}{n !}(i k)^{n},
$$

where $\alpha \neq 1,2,3 \ldots,\left|\ln \left(e^{i k}\right)\right|<2 \pi$. Substituting above equation in Eq. (20) and after few steps of simplification, we get

$$
\begin{aligned}
\chi_{y}= & 2 \lambda\left(\Gamma[1-\alpha](k)^{\alpha-1} \cos \left(\frac{\pi \alpha}{2}\right)\right. \\
& \left.+\sum_{n=0}^{\infty} \frac{\zeta[\alpha-n]}{n !}(k)^{n} \sin \left(\frac{\pi n}{2}\right)\right),
\end{aligned}
$$

Thus the $\chi_{y}$ series diverges if $k \rightarrow 0$ as $(k)^{\alpha-1}$ for all $\alpha<$ 1 and convergence for $\alpha>1$ [Fig. 10(c)]. When $\alpha=1$, the polylogarithmic series expansion in Eq. (22) is ill-defined as per Eq. (21) [53].

The physics becomes even more rich in case of $\mathrm{Ki}$ taev chain with long-range pairing and short-range hopping $[23,26]$. Because of the conditions imposed, one can get new quasiparticles like massive edge (when $\mathbf{M} \neq \mathbf{M}_{c}$ ) modes which are neither zero modes nor can be absorbed by bulk modes. The associated Majorana zero modes are created by $\chi_{y}$ term $\left(\mathbf{M} \rightarrow \mathbf{M}_{c}\right)$ and the massive edge modes are controlled by $\chi_{z}$ term (which is short-range) [26]. These massive edge modes are not prominent in our isotropic Kitaev chain (longrange hopping and long-range pairing) as compared to the case of long-range pairing and short-range hopping. However there are similar works which predict the signature of massive edge modes along with Majorana zero modes for $\alpha<1$ region with different approach [26].

As per the known techniques of momentum space characterization, it is not possible to define the topological invariant in gapless regions as well as $\alpha \leqslant 1$ region of a long-range model because of the nonanalyticity of the Berry connection. In the limit $\alpha>1$, the long-range model reduces to the short range version with a Majorana zero mode at each end while in the limit $\alpha \leqslant 1$, there exists an ill-defined region where the BC acquires nonanalytic form [Fig. 10(d)]. Thus the critical exponents of characteristic length as well as susceptibility factors become undefined in this regime.

\section{A REPRESENTATION OF CRITICALITY THROUGH PARAMETER SPACE}

Here we consider different parameter space and try to analyze the topological phases as well as TQPTs through pseudospins as well as from exact calculations.

\section{A. Pseudospin vector parameter space}

Another way to understand the topological properties of the system is through the analysis of parameter space [30,54]. The pseudospin vector $\vec{\chi}\left(\chi_{x}, \chi_{y}, \chi_{z}\right)$ [see Eq. (5)] can be used to study the parameter space. The pseudospin vector components form a closed loop in the parameter space due to the periodic boundary condition. If the closed loop encloses the origin, it represents a topological state. The number of around the origin gives the topological index $W$. If the closed curve does not include the origin, it represents nontopological state. When the curve touches the origin, it is the critical case where TQPT occurs. by

For $r=2$, the components of pseudospin vector are given

$$
\begin{aligned}
& \chi_{x}=0, \quad \chi_{y}=2 \lambda\left(\sin (k)+\frac{\sin (2 k)}{2^{\alpha}}\right), \\
& \chi_{z}=-\mu-2 \lambda\left(\cos (k)+\frac{\cos (2 k)}{2^{\alpha}}\right) .
\end{aligned}
$$

Figure 11 represents the pseudospin vector in the parameter space for $r=2$. We observe a transition between $W: 0 \leftrightarrow 1$ for different values of $\alpha$. The upper panel represents the case when $\alpha=0$. Here we observe transition among $W: 0 \leftrightarrow 2$ through a TQPT [Fig. 11(b)] followed by a transition between $W: 2 \leftrightarrow 1$ through another TQPT [Fig. 11(d)]. Also we observe a direct transition between $W: 1 \leftrightarrow 0$ trough a third TQPT. We note that for all the TQPTs the closed curve touches the origin. The lower panel represents $\alpha=1$ case. Here we observe a direct transition between $W: 0 \leftrightarrow 1$ only as we do not find any $W=2$ phase.

When the pseudospin curves touch the origin, it represent TQPTs. In general, BC becomes nonanalytic at these points and WN becomes ill-defined. However, there are efforts which show the localized edge modes even at the gapless region [36,38,41-47] especially in longer-range models $[37,39,40,48,49]$.

Here we use Eq. (8) to find the topological invariant around criticality by taking the limit $k \rightarrow k_{0}$ as shown in Table III. We can also observe similar nature in $r=3$ and $r=4$ cases. This is because with the increasing values of $\alpha$, a longerrange model reduces to original Kitaev chain which we have observed also from previous sections.

This analysis can also be generalized to a long-range model with some modifications. For a one-dimensional system with periodic boundary conditions, the polylogarithmic functions take the form

$$
L i_{\alpha}[z]=\sum_{l=1}^{\infty} \frac{z^{l}}{l^{\alpha}} .
$$

which is the polylogarithm of complex function $z$ with order $\alpha$ and for our model $z=e^{i k} \Longrightarrow|z|=1$. Substituting it in 


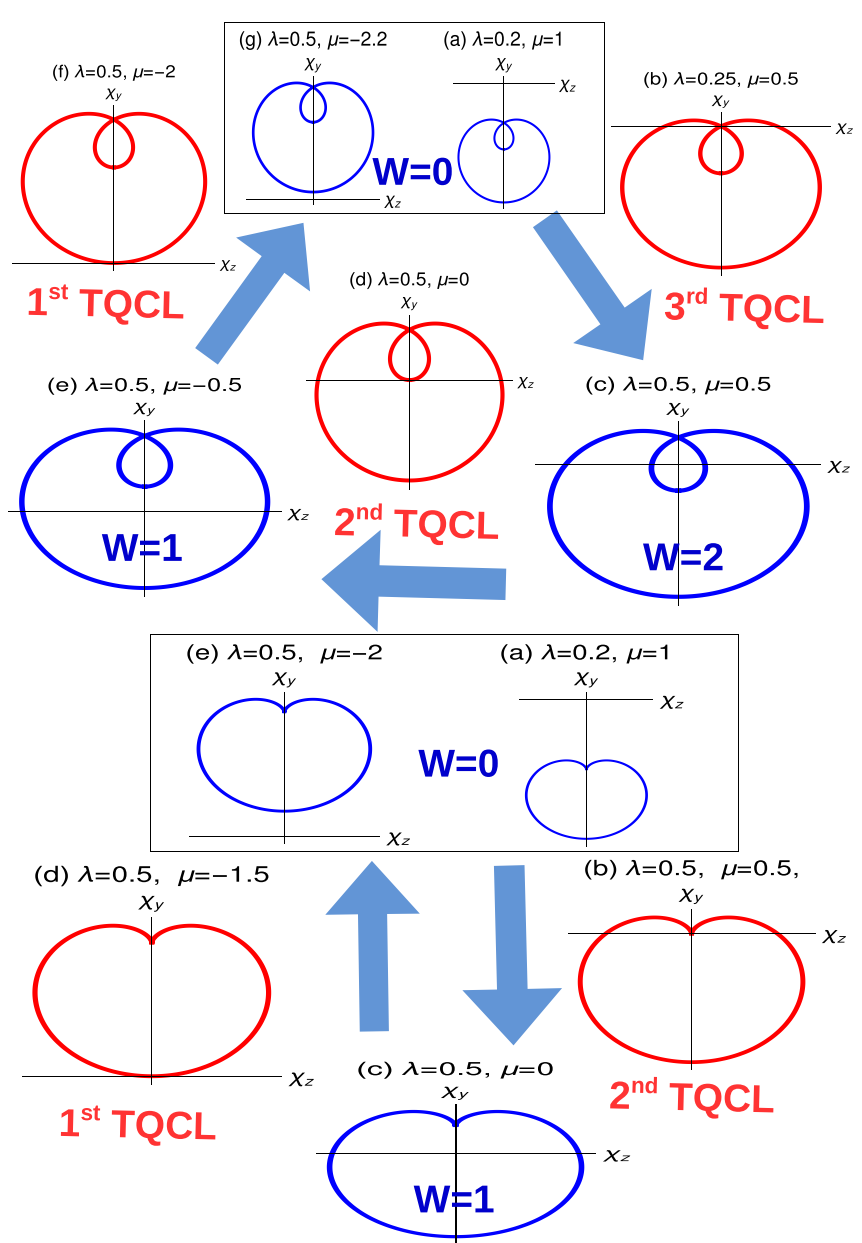

FIG. 11. Closed curves representing the pseudospin vectors for different values of parameters and for $r=2$. Blue and red curves are topological phases and TQPT, respectively. (Top) $(\alpha=0)$ : the longer-range Kitaev chain exhibits TQPT among $W: 0 \leftrightarrow 2$ through the third TQCL, $W: 2 \leftrightarrow 1$ through the second TQCL and $W: 1 \leftrightarrow$ 0 through the first TQCL. (Bottom) $(\alpha=1)$ : the reduced longerrange Kitaev chain exhibits only two TQPTs. From $W: 0 \leftrightarrow 1$ through the second TQCL and $W: 1 \leftrightarrow 0$ through the first TQCL.
Eq. (20), one can get the pseudospin parameters in terms of complex function as shown in Fig. 12.

It is clear from the analysis of Sec. IV D, that the $\alpha>1$ is the topological regime, where the pseudospin vector takes a complete rotation around the origin, i.e., $W=1$ [Fig. 12(a)]. For the nontopological phase, i.e., $W=0$, the pseudospin vector fails to take a rotation around the origin [Fig. 12(b)]. The region $\alpha<1$ is ill-defined from the topological perspective, where we find non-closing curves [Fig. 12(c)]. The point $\alpha=1$ represents a phase transition without gap closing, hence we find a closed curve which does not touches the origin [Fig. 12(d)]. One can obtain the fractional and integer winding numbers for $\alpha<1$ and $\alpha>1$ regime respectively. The point $\alpha=1$ is a case where the pseudospin vectors are neither convergent nor divergent [Fig. 10(c)]. Thus we find a closed curve which contains less populated vectors around $k=0$, where the curvature function still behaves as nonanalytic resulting in the fractional WN. We observe same behavior of closed curve and less population of vectors around $k=0$ till $\alpha=1.5$. But this regime contains a nondivergent curvature function along with integer winding number. The authors of Ref. [27] have used a similar approach of pseudospin vector curves to characterize the topological phases in a similar model. Also fractional winding number has been obtained in Ref. [26] in the same regime for a very similar model.

\section{B. A few exact solutions for topological characterization}

Here we try to find some exact solutions of the winding number for different parameters. It is difficult to find exact solutions for the parameter spaces as the associated integral may become really complicated. We consider only some of the special cases and present the exact solutions of $\mathrm{WN}$ for $r=2$ only. Equation (9) gives the expression of $\mathrm{WN}$ for $r=$ 2. WN always gives integer number for gapped topological phases. However, for gapless phases, WN may take integer or fraction values depending on the parameter space. For a gapless phase we calculate $\mathrm{WN}$ by omitting the gap closing points, i.e., $k=0, \pi$ and $\cos ^{-1}\left(-2^{\alpha-1}\right)$. Here the $\mathrm{WN}$ takes

TABLE III. A few exact solutions for the winding number when $r=2$.

\begin{tabular}{|c|c|c|c|c|}
\hline $\begin{array}{l}\text { first TQCL }(k=0) \\
\left(\mu=-2 \lambda\left(1+1 / 2^{\alpha}\right)\right)\end{array}$ & 0 & $\mu=-4 \lambda$ & $W=\left(\frac{1}{2 \pi}\right) \int_{-\pi}^{\pi} \frac{7+8 \cos (k)}{10+8 \cos (k)} d k$ & $\mathrm{~W}=1 / 2$ \\
\hline \multirow{2}{*}{$\begin{array}{l}\text { second TQCL }(k=\pi) \\
\left(\mu=-2 \lambda\left(-1+1 / 2^{\alpha}\right)\right)\end{array}$} & 0 & $\mu=0$ & $W=\left(\frac{1}{2 \pi}\right) \int_{-\pi}^{\pi} 3 / 2 d k$ & $\mathrm{~W}=3 / 2$ \\
\hline & 1 & $\mu=\lambda$ & $W=\left(\frac{1}{2 \pi}\right) \int_{-\pi}^{\pi} d k$ & $\mathrm{~W}=1$ \\
\hline $\begin{array}{l}\text { third TQCL }\left(k=\cos ^{-1}\left(-2^{\alpha-1}\right)\right) \\
\left(\mu=\lambda / 2^{\alpha-1}\right)\end{array}$ & 1 & $\mu=\lambda$ & $W=\left(\frac{1}{2 \pi}\right) \int_{-\pi}^{\pi} d k$ & $\mathrm{~W}=1$ \\
\hline Gapped phase & 0 & $\mu=-\lambda$ & $W=\lim _{\delta \longrightarrow 0^{+}}\left(\frac{1}{2 \pi}\right) \int_{\pi-\delta}^{\pi+\delta} \frac{3+6 \cos (k)}{9+4 \cos (k)-4 \cos (2 k)} d k$ & $\mathrm{~W}=1$ \\
\hline Gapped phase & 1 & $\mu=-\lambda$ & $W=\lim _{\delta \rightarrow 0^{+}}\left(\frac{1}{2 \pi}\right) \int_{\pi-\delta}^{\pi+\delta} 1-\frac{2 \cos (k)}{-3+\cos (2 k)} d k$ & $\mathrm{~W}=1$ \\
\hline Gapped phase & 0 & $\mu=\lambda$ & $W=\lim _{\delta \longrightarrow 0^{+}}\left(\frac{1}{2 \pi}\right) \int_{\pi-\delta}^{\pi+\delta} \frac{3+2 \cos (k)}{9+12 \cos (k)+4 \cos (2 k)} d k$ & $\mathrm{~W}=2$ \\
\hline
\end{tabular}




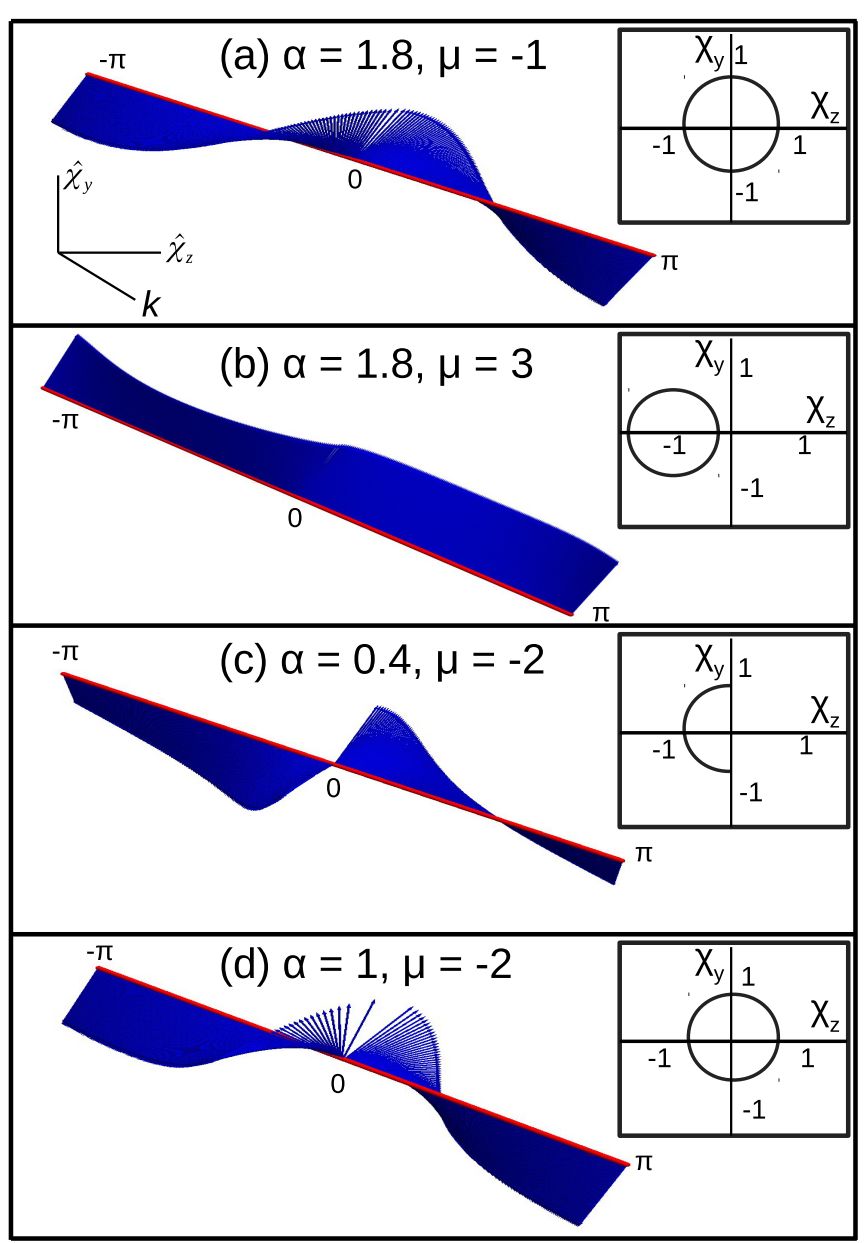

FIG. 12. Pseudospin parameter (unit vector) plots for different regimes of $\alpha, \mu$ with $\lambda=1$ and system size $l=200$. (a) For $\alpha>1$, the parameter curve completes a full circle around the $k$ axis. (b) For $\alpha>1$, the curve does not completes a circle around the $k$ axis. (c) For $\alpha<1$, the curve shows a discontinuity around $k=0$. (d) For $\alpha=1$, the curve completes a full circle, but contains less populated winding vectors around $k=0$. (Inset) Corresponding two-dimensional representation of parameter plots.

the form

$$
W=\int \frac{A+B \cos (k)+C \cos (2 k)}{a+b \cos (k)+c \cos (2 k)},
$$

where

$$
\begin{aligned}
& A=4 \lambda^{2}\left(2+2^{2 \alpha}\right), B=2^{\alpha+1} \lambda\left(6 \lambda+2^{\alpha} \mu\right), \\
& C=c=2^{\alpha+2} \lambda \mu, a=4 \lambda^{2}\left(1+2^{\alpha}\right) 2^{2 \alpha} \mu^{2}, \\
& b=2^{\alpha+2} \lambda\left(2^{\alpha} \mu+2 \lambda^{2}\right) .
\end{aligned}
$$

Based on parameter space, WN takes different standard integral formats $[16,30,53]$.

Some of the standard integral formats are

(1) $\int \frac{A+B \cos k}{a+b \cos k} d k$ when $a^{2}>b^{2}(C=c=0)$

$$
=\frac{B}{b} k+\frac{2(A b-a B)}{b \sqrt{a^{2}-b^{2}}} \tan ^{-1}\left(\frac{\sqrt{a^{2}-b^{2}} \tan k / 2}{a+b}\right) ;
$$

(2) $\int \frac{A+B \cos k}{a+b \cos k} d k$ when $b^{2}>a^{2}(C=c=0)$

$$
=\frac{B}{b} k+\frac{2(A b-a B)}{b \sqrt{b^{2}-a^{2}}} \ln \left(\frac{\sqrt{b^{2}-a^{2}} \tan k / 2+(a+b)}{\sqrt{b^{2}-a^{2}} \tan k / 2-(a+b)}\right) .
$$

Other than this we have couple of more cases.

(3) Consider $z=\exp (i k)$ and $d k=d z / i z$,

$$
\begin{aligned}
\int \frac{A d k}{a+b \cos (k)} & =A \oint \frac{d z / i z}{a+b(z+1 / z) / 2} \\
& =8 \pi A\left(z_{+}-z_{-}\right) / b
\end{aligned}
$$

where $z_{ \pm}=-a / b \pm \sqrt{a^{2} / b^{2}-1}$. Here $z_{+}\left(z_{-}\right)$is defined as the root that is inside (outside) the contour $|z|=1$.

(4) In the similar way,

$$
\int \frac{B \cos (k) d k}{a+b \cos (k)}=\left(\frac{z_{+}}{z_{+}-z_{-}}+\frac{1}{z_{+} z_{-}}+\frac{1}{z_{+}\left(z_{+}-z_{-}\right)}\right) .
$$

Based on parameter space, WN gets different quantized values. Here we consider a few cases (see Table III).

For a topological system, WN represents the number localized edge modes of the gapped phases. Based on the number of interacting neighbors $r$, here we get corresponding exact solutions for the topological phases. However, at the criticality, the exact solutions are calculated by omitting gap closing points. For the case $r=2$, the first and third TQCLs give fractional as well as integer exact solutions respectively for all parameter space. The second TQCL gives fractional solutions for initial values and integer solution as $\alpha \rightarrow \infty$ (Table III).

\section{OUTLOOK AND EXPERIMENTAL POSSIBILITIES}

In this work, we have used the isotropic conditions ( $\alpha=\beta$, $\left.J_{0}=\Delta_{0}=\lambda\right)$ and longer-range as well as long-range model to explain topological characterization and criticality of the model. The results of our work can be generalized to other parameter space also. When $\alpha, \beta \rightarrow \infty$, the model reduces to original Kitaev model. But through our work we realize that, the reduction should undergo through the process of superposition and vanishing of TQCL as discussed earlier. According to the available literature, it is clear that the winding number is not enough to understand the topological properties of a long-range model. And there are two different arguments about the reduction of the long-range model to the short-range model. Some works suggest that the reduction happens when $\alpha>1[25,55,56]$ and some studies obtain a short-range limit for $\alpha>1.5$ [27]. Solving these issues through the study of universality class of critical exponents and CFT can give the better understanding. When the pairing term decays slower than hopping parameter, there may be possibilities of obtaining new exotic particles like massive Majorana modes [57]. The results of criticality and behavior of TQCLs may be interesting in those cases.

Experimental possibilities. There are a number of experiments which explore the properties of long-range models especially in trapped ions [58-61], atom coupled to multimode cavities [62], magnetic impurities [63,64], and quantum computation [18]. In long-range models, the characteristic length shrinks for the longer neighbors. Hence, even by using 
a relatively small number of ions it is possible to suppress the finite-size effects [65]. Within the tight-binding BdG formalism, the Shiba chains can be modeled to $p$-wave superconducting Kitaev chain with long-range pairing and hopping $[66,67]$. Naturally Shiba chains exhibits $1 / r$ decay away from certain limits of coherence length [65]. Hence it is easy to map our isotropic Kitaev model in such systems. Analysis of criticality studied in this work may help to explore the subject in a better way.

\section{CONCLUSION}

To summarize, we have presented a theoretical study of the topological quantum phase transitions and quantum criticality in the longer-range as well as long-range Kitaev chain. Here all possible topological criticality conditions have been calculated in detail along with precise topological phase diagram. For a longer-range model, with the increasing number of interacting neighbors, higher order winding numbers have been generated and their stability decreases with the increasing value of the decay parameter, which has been verified by the analysis of ground-state energy. A decrease of winding number has been observed with decreasing long rangeness in the system with a pattern of odd-to-odd and even-to-even transition among winding numbers. As a reason behind this, we show a mechanism of the superposition of two critical lines and vanishing of the one with higher winding number. We have analyzed different possibilities of superposition and different means of vanishing of critical lines. Through this we have studied the nature of multicritical points in the longer-range model. The criticality has been studied from the perspective of critical exponents and their fate near the multicritical points have been analyzed. As the generalization of this work, we have considered a truly long-range model and its momentum space characterization been done. We have analyzed the nonanalytic behavior of Berry connection in both long-range Kitaev chain and longer-range (finite-range) Kitaev chain to study the topological invariant. A parameter space representation is done for longer-range as well as longrange models along with a few exact solutions for the winding numbers in support of our findings. We have also discussed the possible outlook and experimental aspects of our work. Instances of study of topological quantum phase transitions covering long-range models and quantum criticality are rare in the literature. We hope that our work will help boost the understanding of such systems.

\section{ACKNOWLEDGMENTS}

S.S. would like to acknowledge DST (Department of Science and Technology, Government of India) (EMR/2017/000898) for the funding and RRI and ICTS library for the books and journals. N.R. is grateful to the University Grants Commission (UGC), India for providing a PhD fellowship. Y.R.K. would like to thank Admar Mutt Education Foundation for the scholarship. The authors would like to acknowledge B S Ramachandra, Prabir K. Mukherjee, and C Sivaram who read this manuscript critically and gave useful suggestions.

\section{APPENDIX: DETAILED DERIVATION OF CRITICAL LINES FOR LONGER-RANGE KITAEV CHAIN}

Here we derive the topological quantum critical lines of the longer-range Kitaev chain for interacting neighbors $r=2,3$, and 4 . We follow the standard method to find the critical lines. First we find the value of $k_{0}$ by making the term $\chi_{y}=0$. After that we substitute the value of $k_{0}$ into $\chi_{z}=0$ to find the critical line. Depending on the number of interacting neighbors, that many critical lines will be generated.

\section{When $r=2$}

Here the gap closing occurs for three different values of $k$, i.e..

$$
\begin{aligned}
\text { 1) } & k=0 \\
\text { 2) } \quad k & =\pi \\
\text { 3) } \quad k & =\cos ^{-1}\left(-2^{\alpha-1}\right) .
\end{aligned}
$$

Corresponding TQCLs are

$$
\begin{aligned}
& \text { 1) } \mu=-2 \lambda\left(\frac{1}{2^{\alpha}}+1\right) \text {, } \\
& \text { 2) } \mu=-2 \lambda\left(\frac{1}{2^{\alpha}}-1\right) \text {, } \\
& \text { 3) } \mu=\frac{\lambda}{2^{\alpha-1}},
\end{aligned}
$$

for third TQCL $0<\alpha \leqslant 1$.

\section{When $r=3$}

Here the gap closing occurs for four different values of $k$, i.e.,

1) $k=0$,

2) $k=\pi$

3) $k=\cos ^{-1}\left(\frac{3^{\alpha}}{4}\left(-\frac{1}{2}+\sqrt{\frac{1}{2^{\alpha}}-\frac{4\left(3^{\alpha}-1\right)}{3^{2 \alpha}}}\right)\right)$,

4) $k=\cos ^{-1}\left(\frac{3^{\alpha}}{4}\left(-\frac{1}{2}-\sqrt{\frac{1}{2^{\alpha}}-\frac{4\left(3^{\alpha}-1\right)}{3^{2 \alpha}}}\right)\right)$.

Corresponding TQCLs are

$$
\begin{aligned}
& \text { 1) } \mu=-2 \lambda\left(\frac{1}{3^{\alpha}}+\frac{1}{2^{\alpha}}+1\right), \\
& \text { 2) } \mu=-2 \lambda\left(-\frac{1}{3^{\alpha}}+\frac{1}{2^{\alpha}}-1\right), \\
& \text { 3) } \mu=-\lambda\left(-8^{-\alpha-1} Q\right), \\
& \text { 4) } \mu=-\lambda\left(8^{-\alpha-1} R\right),
\end{aligned}
$$

where

$$
\begin{aligned}
& P=\sqrt{2^{-\alpha}-4 \times 9^{-\alpha}\left(3^{\alpha}-1\right)}, \\
& Q=\left(18^{\alpha} P-36^{\alpha} P+8^{\alpha+1} P+9^{\alpha}-18^{\alpha}-2^{2 \alpha+3}\right), \\
& R=\left(18^{\alpha} P-36^{\alpha} P+8^{\alpha+1} P-9^{\alpha}+18^{\alpha}+2^{2 \alpha+3}\right) .
\end{aligned}
$$




\section{When $r=4$} i.e.,

Here the gap closing occurs for five different values of $k$,

$$
\begin{aligned}
& \text { 1) } k=0, \\
& \text { 2) } k=\pi, \\
& \text { 3) } \quad \cos (k)=-2^{2 \alpha-1} \times 3^{-\alpha-1} \\
& +\frac{1}{3} 2^{2 \alpha-\frac{10}{3}} \sqrt[3]{\sqrt{4 A^{3}+X^{2}}+X} \\
& -\frac{2^{2 \alpha-\frac{8}{3}} A}{3 \sqrt[3]{\sqrt{4 A^{3}+X^{2}}+X}}, \\
& \text { 4) } \cos (k)=-2^{2 \alpha-1} \times 3^{-\alpha-1} \\
& -\frac{1}{3}(1-i \sqrt{3}) 2^{2 \alpha-\frac{13}{3}} \sqrt[3]{\sqrt{4 A^{3}+X^{2}}+X} \\
& +\frac{(1+i \sqrt{3}) 2^{2 \alpha-\frac{11}{3}} A}{3 \sqrt[3]{\sqrt{4 A^{3}+X^{2}}+X}}, \\
& \text { 5) } \cos (k)=-2^{2 \alpha-1} \times 3^{-\alpha-1} \\
& -\frac{1}{3}(1+i \sqrt{3}) 2^{2 \alpha-\frac{13}{3}} \sqrt[3]{\sqrt{4 A^{3}+X^{2}}+X} \\
& +\frac{(1-i \sqrt{3}) 2^{2 \alpha-\frac{11}{3}} A}{3 \sqrt[3]{\sqrt{4 A^{3}+X^{2}}+X}},
\end{aligned}
$$

where

$$
\begin{aligned}
A= & 3 \times 2^{4-4 \alpha}\left(2^{\alpha}-2\right)-16 \times 3^{-2 \alpha} \\
X= & -27 \times 2^{6-4 \alpha}+2^{6-4 \alpha} 3^{2-\alpha}+2^{6-3 \alpha} 3^{2-\alpha} \\
& -128 \times 3^{-3 \alpha}
\end{aligned}
$$

$$
\begin{aligned}
P= & \sqrt{4 A^{3}+X^{2}}+X \\
Q= & -2^{2 \alpha-1} 3^{-\alpha-1}-\frac{2^{2 \alpha-\frac{8}{3}} A}{3 \sqrt[3]{P}}+\frac{1}{3} 2^{2 \alpha-\frac{10}{3}} \sqrt[3]{P}, \\
R= & -2^{2 \alpha-1} 3^{-\alpha-1}+\frac{(1-i \sqrt{3}) 2^{2 \alpha-\frac{11}{3}} A}{3 \sqrt[3]{P}} \\
& -\frac{1}{3}(1+i \sqrt{3}) 2^{2 \alpha-\frac{13}{3}} P, \\
S= & -2^{2 \alpha-1} 3^{-\alpha-1}+\frac{(1+i \sqrt{3}) 2^{2 \alpha-\frac{11}{3}} A}{3 \sqrt[3]{P}} \\
& -\frac{1}{3}(1-i \sqrt{3}) 2^{2 \alpha-\frac{13}{3}} P .
\end{aligned}
$$

Corresponding TQCLs are

$$
\text { 1) } \begin{aligned}
\mu= & -2 \lambda\left(\frac{1}{3^{\alpha}}+\frac{1}{4^{\alpha}}+\frac{1}{2^{\alpha}}+1\right), \\
\text { 2) } \quad \mu= & -2 \lambda\left(-\frac{1}{3^{\alpha}}+\frac{1}{4^{\alpha}}+\frac{1}{2^{\alpha}}-1\right), \\
\text { 3) } \quad \mu= & -2 \lambda\left(-2^{1-\alpha}+4^{-\alpha}+2^{3-2 \alpha} Q^{4}\right) \\
& -2 \lambda\left(4 \times 3^{-\alpha} Q^{3}+\left(2^{1-\alpha}-2^{3-2 \alpha}\right) Q^{2}\right. \\
& \left.+\left(1-3^{1-\alpha}\right) Q\right), \\
4= & -2 \lambda\left(-2^{1-\alpha}+4^{-\alpha}+2^{3-2 \alpha} S^{4}+4 \times 3^{-\alpha} S^{3}\right) \\
& -2 \lambda\left(\left(2^{1-\alpha}-2^{3-2 \alpha}\right) S^{2}+\left(1-3^{1-\alpha}\right) S\right), \\
\text { 5) } \quad & -2 \lambda\left(-2^{1-\alpha}+4^{-\alpha}+2^{3-2 \alpha} R^{4}+4 \times 3^{-\alpha} R^{3}\right) \\
& -2 \lambda\left(\left(2^{1-\alpha}-2^{3-2 \alpha}\right) R^{2}+\left(1-3^{1-\alpha}\right) R\right) .
\end{aligned}
$$

Information Theory (Springer, Natal, Brazil, 2020), pp. 289307.

[12] S. Rufo, N. Lopes, M. A. Continentino, and M. A. R. Griffith, Phys. Rev. B 100, 195432 (2019).

[13] S. Sachdev, Quantum phase transitions, Handbook of Magnetism and Advanced Magnetic Materials, edited by Helmut Kronmüller and Stuart Parkin Fundamentals and Theory Vol. 1 (John Wiley and Sons, Ltd., New Jersey, 2007).

[14] R. Kumar R, S. Rahul, S. N. Sahoo, and S. Sarkar, Phase Trans. 93, 606 (2020).

[15] S. Sarkar, Sci. Rep. 10, 1 (2020).

[16] W. Chen and M. Sigrist, Topological phase transitions: Criticality, universality, and renormalization group approach, Advanced Topological Insulators (Wiley Online Library, Hoboken, USA, 2019), Vol. 239.

[17] D. F. Abasto, A. Hamma, and P. Zanardi, Phys. Rev. A 78, 010301(R) (2008).

[18] S. T. Amin, B. Mera, N. Paunković, and V. R. Vieira, J. Phys.: Condens. Matter 31, 485402 (2019).

[19] A. Y. Kitaev, Phys. Usp. 44, 131 (2001).

[20] S. D. Sarma, M. Freedman, and C. Nayak, npj Quantum Inf. 1, 1 (2015).

[21] P. Cats, A. Quelle, O. Viyuela, M. A. Martin-Delgado, and C. M. Smith, Phys. Rev. B 97, 121106(R) (2018). 
[22] Q.-J. Tong, J.-H. An, J. Gong, H.-G. Luo, and C. H. Oh, Phys. Rev. B 87, 201109 (2013).

[23] D. Vodola, L. Lepori, E. Ercolessi, A. V. Gorshkov, and G. Pupillo, Phys. Rev. Lett. 113, 156402 (2014).

[24] O. Viyuela, L. Fu, and M. A. Martin-Delgado, Phys. Rev. Lett. 120, 017001 (2018).

[25] L. Lepori, D. Giuliano, and S. Paganelli, Phys. Rev. B 97, 041109(R) (2018).

[26] A. Alecce and L. Dell'Anna, Phys. Rev. B 95, 195160 (2017).

[27] O. Viyuela, D. Vodola, G. Pupillo, and M. A. Martin-Delgado, Phys. Rev. B 94, 125121 (2016).

[28] Y. Niu, S. B. Chung, C.-H. Hsu, I. Mandal, S. Raghu, and S. Chakravarty, Phys. Rev. B 85, 035110 (2012).

[29] P. W. Anderson, Phys. Rev. 110, 827 (1958).

[30] S. Sarkar, Sci. Rep. 8, 5864 (2018).

[31] F. Wilczek and A. Shapere, Geometric Phases in Physics (World Scientific, Singapore, 1989), Vol. 5.

[32] M. Berry, J. Phys. A: Math. Gen. 18, 15 (1985).

[33] S. Kempkes, A. Quelle, and C. M. Smith, Sci. Rep. 6, 38530 (2016).

[34] S. Chen, L. Wang, Y. Hao, and Y. Wang, Phys. Rev. A 77, 032111 (2008).

[35] I. Mahyaeh and E. Ardonne, J. Phys. Commun. 2, 045010 (2018).

[36] N. G. Jones and R. Verresen, J. Stat. Phys. 175, 1164 (2019).

[37] R. Verresen, R. Thorngren, N. G. Jones, and F. Pollmann, arXiv:1905.06969.

[38] R. Thorngren, A. Vishwanath, and R. Verresen, arXiv:2008.06638.

[39] R. Verresen, arXiv:2003.05453.

[40] R. Verresen, N. G. Jones, and F. Pollmann, Phys. Rev. Lett. 120, 057001 (2018).

[41] J. P. Kestner, B. Wang, J. D. Sau, and S. Das Sarma, Phys. Rev. B 83, 174409 (2011).

[42] M. Cheng and H.-H. Tu, Phys. Rev. B 84, 094503 (2011).

[43] L. Fidkowski, R. Lutchyn, C. Nayak, and M. Fisher, Phys. Rev. B. 84, 195436 (2011).

[44] J. D. Sau, B. I. Halperin, K. Flensberg, and S. Das Sarma, Phys. Rev. B 84, 144509 (2011).

[45] C. V. Kraus, M. Dalmonte, M. A. Baranov, A. M. Läuchli, and P. Zoller, Majorana Edge States in Atomic Wires Coupled by Pair Hopping, Phys. Rev. Lett. 111, 173004 (2013).

[46] T. Scaffidi, D. E. Parker, and R. Vasseur, Phys. Rev. X 7, 041048 (2017).
[47] H.-C. Jiang, Z.-X. Li, A. Seidel, and D.-H. Lee, Sci. Bulletin 63, 753 (2018).

[48] R. R. Kumar, Y. R. Kartik, S. Rahul, and S. Sarkar, Sci. Rep. 11, 1 (2021).

[49] S. Rahul, Y. R. Kartik, R. Ranjith, and S. Sarkar, arXiv:1906.04462.

[50] P. Molignini, R. Chitra, and W. Chen, Europhys. Lett. 128, 36001 (2020).

[51] P. Molignini, W. Chen, and R. Chitra, Phys. Rev. B 98, 125129 (2018).

[52] M. Malard, D. Brandao, P. E. de Brito, and H. Johannesson, Phys. Rev. Res. 2, 033246 (2020).

[53] F. W. Olver, D. W. Lozier, R. F. Boisvert, and C. W. Clark, NIST Handbook of Mathematical Functions Hardback and CD-ROM (Cambridge University Press, Cambridge, 2010).

[54] G. Zhang and Z. Song, Phys. Rev. Lett. 115, 177204 (2015).

[55] D. Giuliano, S. Paganelli, and L. Lepori, Phys. Rev. B 97, 155113 (2018).

[56] L. Lepori and L. Dell'Anna, New J. Phys. 19, 103030 (2017).

[57] D. Vodola, L. Lepori, E. Ercolessi, and G. Pupillo, New J. Phys. 18, 015001 (2015).

[58] X.-L. Deng, D. Porras, and J. I. Cirac, Phys. Rev. A 72, 063407 (2005).

[59] J. W. Britton, B. C. Sawyer, A. C. Keith, C.-C. J. Wang, J. K. Freericks, H. Uys, M. J. Biercuk, and J. J. Bollinger, Nature (London) 484, 489 (2012).

[60] P. Hauke, F. M. Cucchietti, A. Müller-Hermes, M.-C. Bañuls, J. I. Cirac, and M. Lewenstein, New J. Phys. 12, 113037 (2010).

[61] N. Roy, A. Sharma, and R. Mukherjee, Phys. Rev. A 99, 052342 (2019).

[62] J. S. Douglas, H. Habibian, C.-L. Hung, A. V. Gorshkov, H. J. Kimble, and D. E. Chang, Nat. Photonics 9, 326 (2015).

[63] K. Zhang, P. Wang, and Z. Song, Sci. Rep. 9, 4978 (2019).

[64] G. C. Ménard, S. Guissart, C. Brun, S. Pons, V. S. Stolyarov, F. Debontridder, M. V. Leclerc, E. Janod, L. Cario, D. Roditchev et al., Nat. Phys. 11, 1013 (2015).

[65] Z.-X. Gong, M. F. Maghrebi, A. Hu, M. L. Wall, M. Foss-Feig, and A. V. Gorshkov, Phys. Rev. B 93, 041102(R) (2016).

[66] J. Röntynen and T. Ojanen, Phys. Rev. Lett. 114, 236803 (2015).

[67] F. Pientka, L. I. Glazman, and F. von Oppen, Phys. Rev. B 89, 180505(R) (2014). 